\title{
FUTURE CINEMA:
}

THE TRANSITION FROM FILM-MAKING TO EXPERIENCE-MAKING EXPERIENCING ALZHEIMER'S THROUGH IMMERSIVE DESIGN FOR EMOTIONS AND EMPATHY IN EXTENDED REALITIES

by

Assem Kroma

\author{
A MRP \\ presented to Ryerson University \\ in partial fulfillment of the \\ requirements for the degree of \\ Master \\ In the Program of \\ Digital Media \\ Toronto, Ontario, Canada, 2018 \\ (C)Assem Kroma, 2018
}




\section{Author's Declaration for Electronic Submission of a MRP}

I hereby declare that I am the sole author of this MRP. This is a true copy of the MRP, including any required final revisions.

I authorize Ryerson University to lend this MRP to other institutions or individuals for the purpose of scholarly research.

I further authorize Ryerson University to reproduce this MRP by photocopying or by other means, in total or in part, at the request of other institutions or individuals for the purpose of scholarly research.

I understand that my MRP may be made electronically available to the public. 
Future Cinema: The Transition from Film-Making to Experience-Making Experiencing Alzheimer's through Immersive Design for Emotions and Empathy in Extended Realities

\author{
Assem Kroma \\ Master of Digital Media \\ Ryerson University, 2018
}

\begin{abstract}
Demographic projections of many western democracies show them to be aging nations. To keep thriving we must ensure that our citizens are aging in a healthy manner without isolation from society. This means, in turn, that we must increase our understanding for the experience of aging in society. Alzheimer's disease is one of the most misunderstood conditions for our aging populations, and is a problematic condition for caregivers and family members to support. This MRP seeks to build extended reality experiences that immerse users emotionally in some of the symptoms of aging and Alzheimer's to build understanding and empathy. The project seeks to use mixed reality to make a positive social impact on the general population.
\end{abstract}




\section{Acknowledgements}

Alzheimer's Eyes Challenge is an Experiential Media Institute project. This MRP wouldn't be possible without the exemplary guidance and supervision of Dr. Richard Lachman. Special thanks to Matt Marshall and Arturo Ortiz for technical advising; and to Dr. Alexandra Bal for the valuable helpful comments and feedback on the previous version of this document. 


\section{Dedication}

To my mother, who gifted me life, who gave up her dreams to gift me mine, and has always been my No. 1 critic/ fan. We did it, mom.

To my dad, on his 15 th anniversary.

To my big sister, my second mom, and my first dancing teacher.

To my big brother, my first acting supporter and my backbone in hard times.

To my little sister, my first best friend and childhood adventures sidekick.

To my wife, my first true love and my partner in crime.

To my $1^{\text {st }}$ grade teacher, I owe you all that I learned, for teaching me the alphabets.

To my filmmaking mentors, for helping me make some artistic dreams come true and supporting me until this very moment.

To my first academic research supervisor, without you, this project would be no more than a dream.

To the MDM program management for betting on me and allowing me to go on this journey.

To my 15 years old self, who would have imagined?! 


\section{Table of Contents}

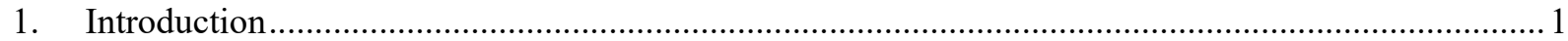

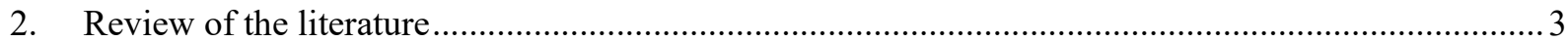

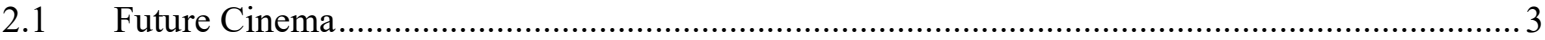

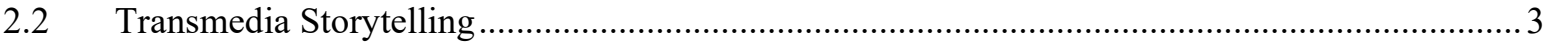

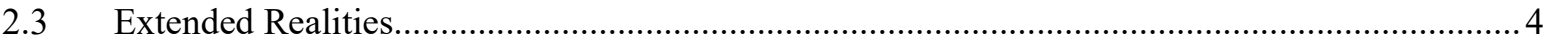

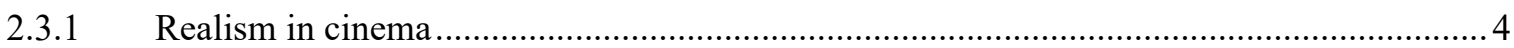

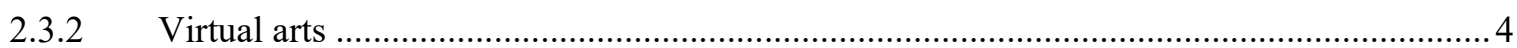

2.3.3 What Constitutes Extended Realities .....................................................................

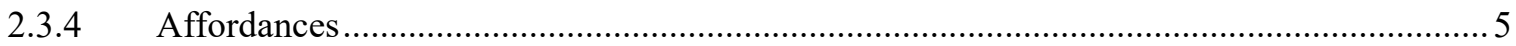

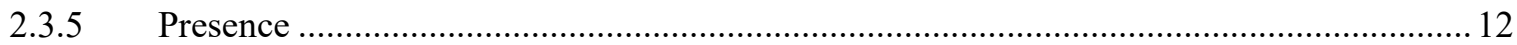

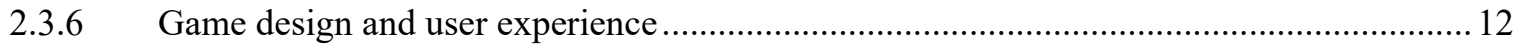

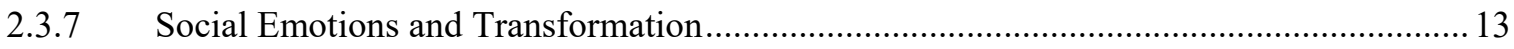

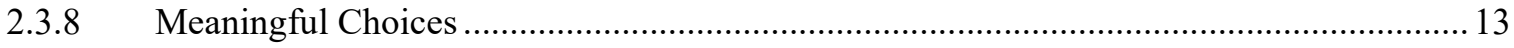

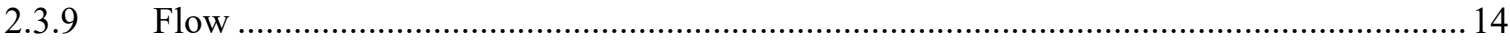

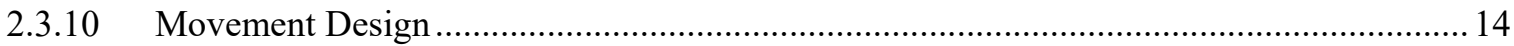

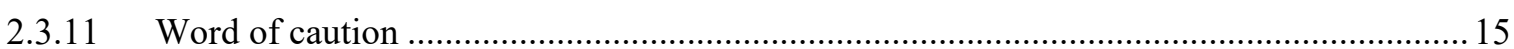

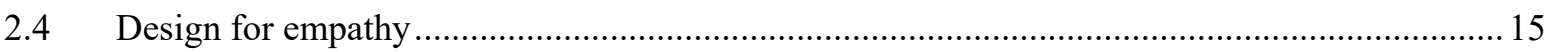

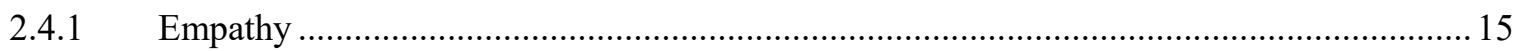

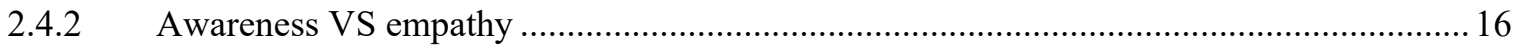

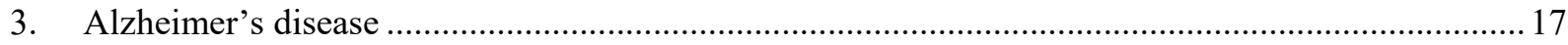

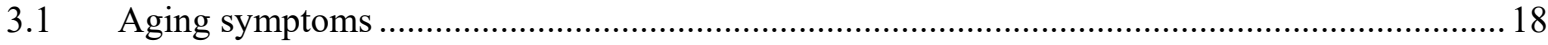

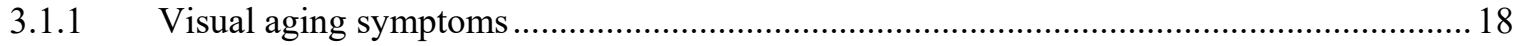

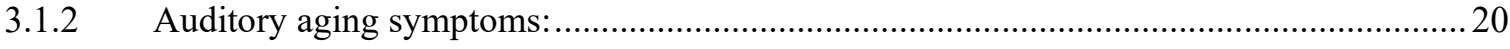

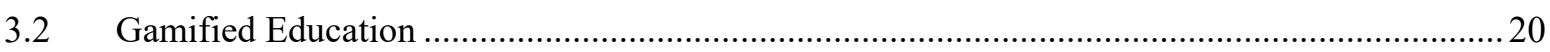

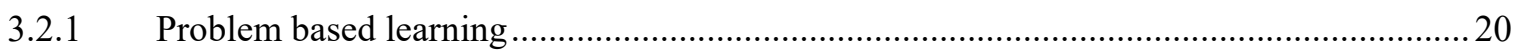

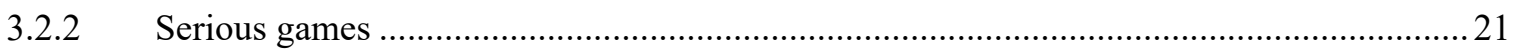

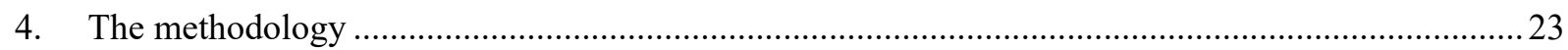

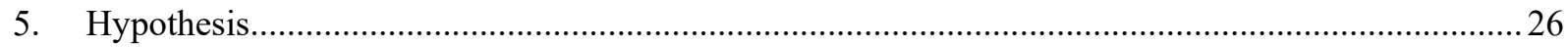

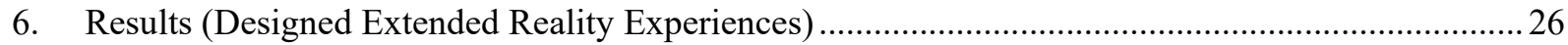

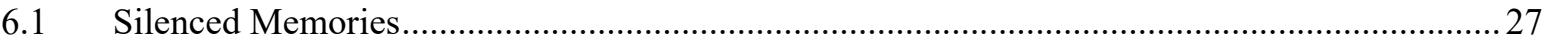

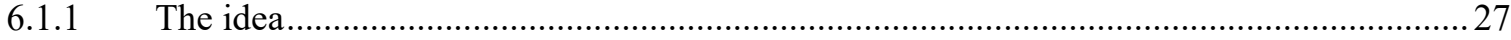




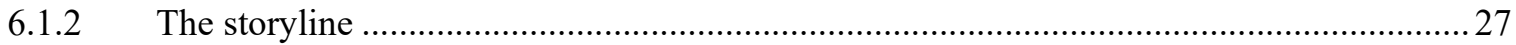

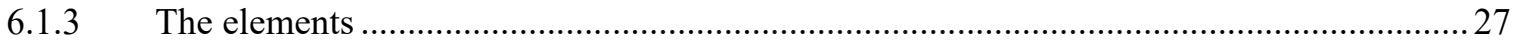

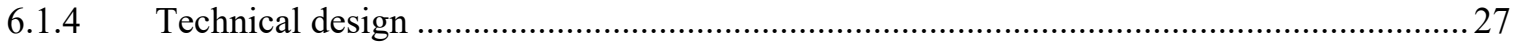

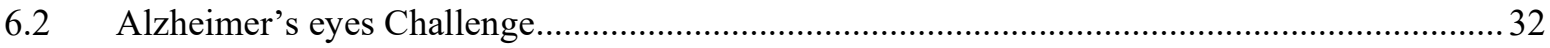

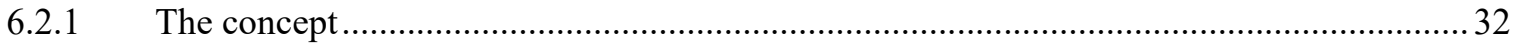

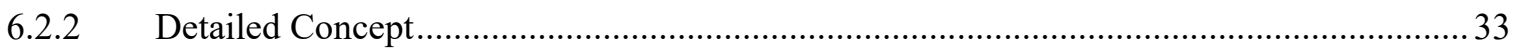

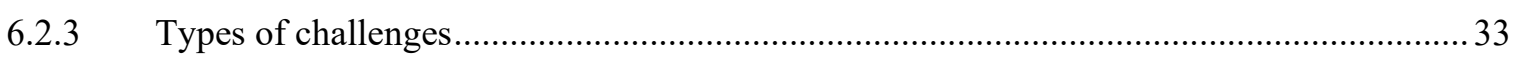

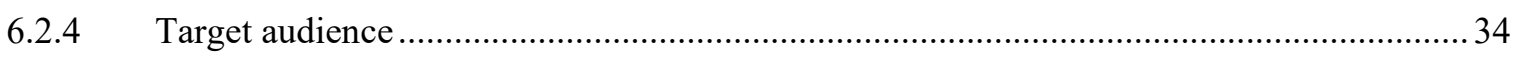

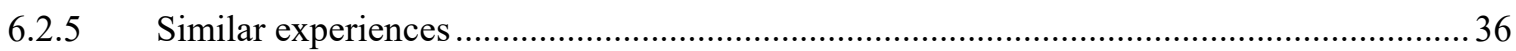

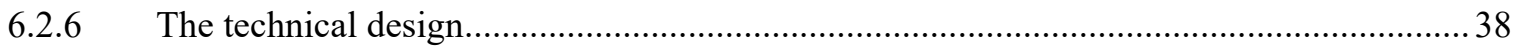

6.2.7 Detailed Description of the first type of the challenge...................................................... 41

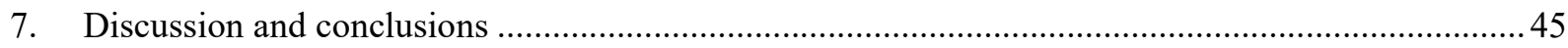

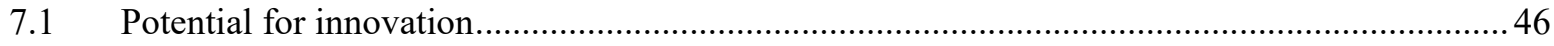

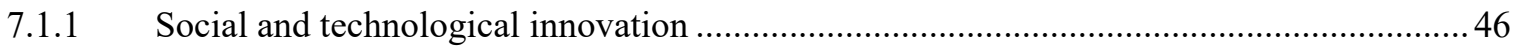

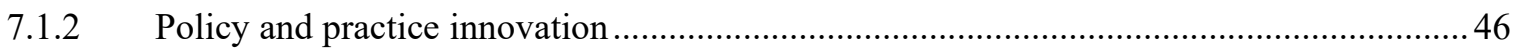

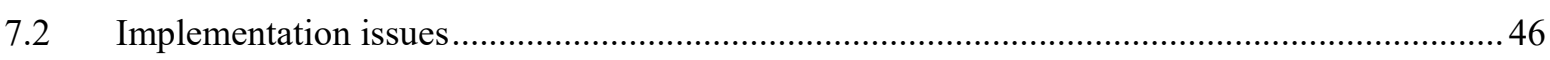

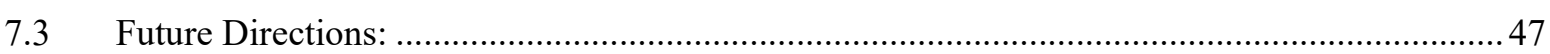

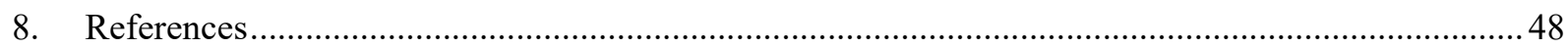




\section{List of Tables}

Table 1: Comparison between different Alzheimer's and Dementia experiences. 


\section{List of Figures}

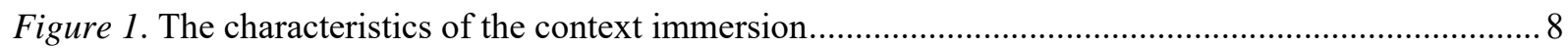

Figure 2. Dimensions of the context immersion in the MR environments............................................... 8

Figure 3. Diagram of a model on immersion, presence, and performance .............................................. 12

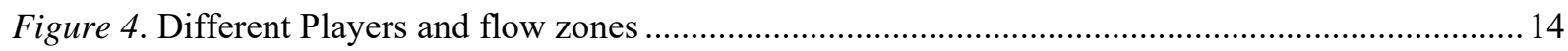

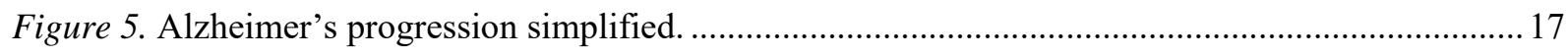

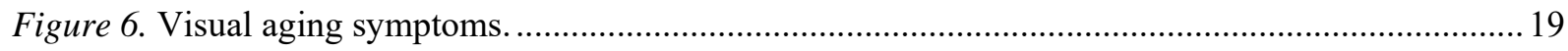

Figure 7. Adapted from the less restrictive variant of the effective-tutor model......................................2

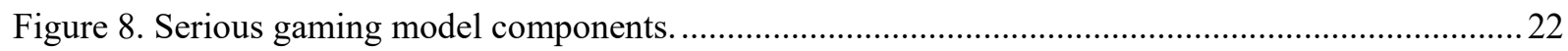

Figure 9. Adapted from the causal model of learning upon engaging in game activity...........................22

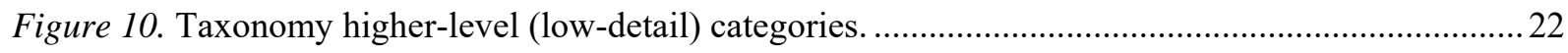

Figure 11. immersive design for emotions and empathy in extended realities.........................................24

Figure 12. A shot from "Silence", a short film by Assem Kroma, to which this experience is

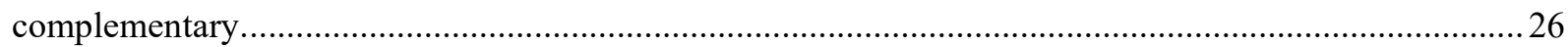

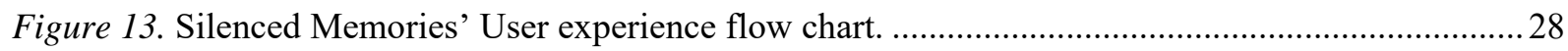

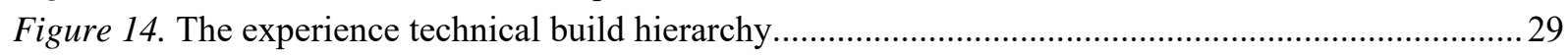

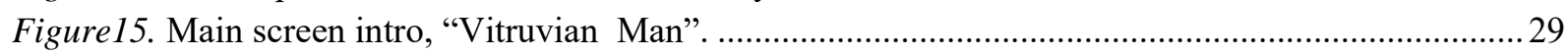

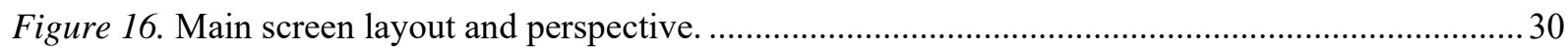

Figure 17. Death tunnel being drawn in the "Temporariness" timeline while the participant hears a

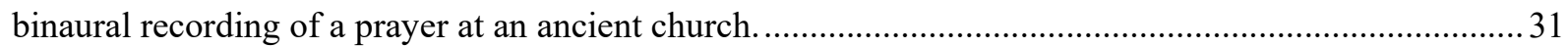

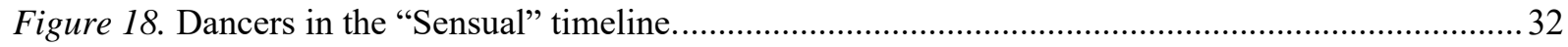

Figure 19. promotional shots taken using the actual experience through the headset................................ 32

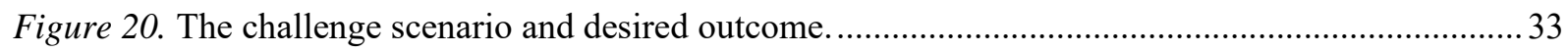

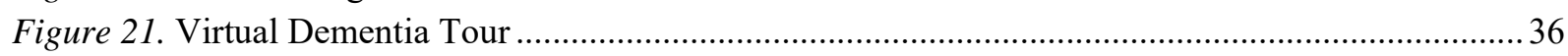

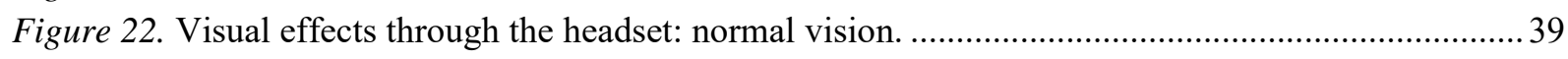

Figure 23. Visual effects through the headset: eye floaters and advanced cataract...................................39

Figure 24. Visual effects through the headset: Macular degeneration and advanced cataract. .................. 40

Figure 25. Visual effects through the headset: stage six, eye floaters and shaking display ……................ 40

Figure 26. Alzheimer's Eyes Challenge User Flow Chart. .................................................................... 42

Figure 27. The exact images which should be recognized by Vuforia to trigger different stages.............. 43 


\section{Introduction}

In future cinema, could we utilize the affordances of extended realities towards a more ethical and socially responsible form of media?

This research project is heavily influenced by Bazin's and Youngblood's work (Bazin \& Andrew, 2014; Youngblood, 1970). They argue that cinema in the future is going to change in nature to transform beyond the frame; they see it becoming interactive and intersected forms of culture. Our storytelling platforms are changing, which, in turn, requires an innovative way of thinking to ensure that these platforms reach their true potential instead of enforcing older outdated techniques on them. Since the start of cinema, filmmakers tried to invent new ways of telling their stories; for example, the Russian filmmaker Sergei Eisenstein introduced different montage techniques as an essential part of the cinematic language (Eisenstein, 1991). These techniques such as "Intellectual Montage" changed the cinema from what it used to be; it allowed filmmakers to create new reality by using two separate shots and placing them creatively together. Eisentstein argued that, by introducing intellectual montage, he made the process not narrative but rather creative (Begin, 2006).

These new platforms include "extended realities" which is a term that refers to virtual, augmented and mixed realities. The participatory nature of extended realities, similarly, is a major factor to consider while utilizing them as storytelling platforms. This project explores the potential of extended realities in one particular socially-relevant context: that of aging.

Statistically, Canada is an aging nation (Data Tables, 2016 Census, 2018), and to keep thriving we have to make sure that our citizens are aging in a healthy manner without being isolated from the rest of the society. Alzheimer's is one of the most misunderstood diseases related to aging, and should be understood within its context and effect on brain health in 
general. This project seeks to build a mixed reality environment to allow participants to experience simulations of Alzheimer's symptoms as part of a multi-stream empathy-building awareness campaign. Although there have been many attempts to simulate Alzheimer's such as "A Walk through Dementia" (2018), the use of flat-screen animation and the lack of social context, in my opinion, reduced the potential impact.

Jenkins (2003) highlighted that there has been a shift in producing content across media to shape a new form of storytelling by delivering unique pieces of content on different platforms to increase engagement, he refers to it by "Transmedia storytelling", to differentiate it from the common concept of "cross media" storytelling. The main focus of this research is to utilize transmedia storytelling concepts to build extended reality experiences around Alzheimer's disease. These experiences utilize design for emotions and empathy to increase immersion and promote social awareness. The successful implementation could be used later as a model for further applications connecting media to ethical and social responsibility, while emphasizing future-cinema's transition to making experiences rather than making films.

One of the main thematic debates of this research is that, as we are transitioning towards future cinema, new technologies are giving us the opportunity to start experiencing films rather than watching them. These new developments should be considered when making films. This consideration would lead to designing a model that utilizes all aspects of experiential and traditional storytelling platforms, to transform the way the audience consume films. In other words, it will start the transition from "film-making" to "experience-making". One of the main differences in this research is that the whole experience design is mainly driven by storytelling and social responsibility; however, this doesn't mean that engagement could not be translated into profit at a later stage by utilizing transmedia storytelling techniques; Bearing in mind that it 
would be designed by the filmmaker, as opposed to a transmedia storyteller who is solely driven by increasing social engagement.

\section{Review of the literature}

\subsection{Future Cinema}

The realist film theorist Andre Bazin predicted that the future of cinema is going to be holographic without boundaries (Bazin \& Andrew, 2014). Gene Youngblood (1970) referred to this transition as "expanded cinema": the transition from frame towards immersive interactive and interconnected forms of culture. In my opinion, we should not apply conventional storytelling techniques on these new platforms. Bauman (2000) believes that these technological developments should be understood within their context (space and time in relation to capitalism) not just through their technological attributes. This research paper focuses on the fact that new technologies and storytelling platforms require new creative approaches that benefit from their true strengths.

\subsection{Transmedia Storytelling}

"Star Wars" is considered one of the best examples of "Transmedia Storytelling". The huge success the first movie achieved made the production house consider new ways of investing that success. Gradually, they increased their fans engagement; they started designing different products for them to consume. They designed games, standalone sub-stories on different platforms, virtual reality experiences, and many other types of consumable content (Jenkins, 2003). In the recent years, the new "Star Wars" films have presented a clear shift in the industry understanding of its fans, to build an increasingly incorporative and collaborative rather than prohibitive strategy. This shift was accompanied by an increase in the industry attempts to understand the dynamics of fans engagement and how to maximize fans loyalty and appreciation (Guynes \& Hassler-Forest, 2017). This developed interest is partially due to the participatory culture that had emerged in 1985 (Delwiche \& Henderson, 2012). 


\subsection{Extended Realities}

It is important to identify several elements to have a clear understanding of extended realities as a concept and platform.

\subsubsection{Realism in cinema}

The idea of realism is not a new one; it started with film as a form of art. While film theorists insisted on the differentiation between film and reality to become a valid art. André Bazin argued against it by stating that its artistic essence is in its ability to reproduce reality and not the other way round. This gave birth to the realist theory. He claimed that the long-take and deep-focus style is the purest form of cinematic realism. Evidently, André Bazin is one of the pioneers in future cinema in his "Myth of Total Cinema" (Bazin \& Andrew, 2014). And it could be argued that virtual, mixed and augmented realities are manifestations of his theories.

\subsubsection{Virtual arts}

Grau (2003) was one of the first art historians who challenged the normal understanding of art history at the time. He linked film to its artistic context in all of its forms, which made the development of extended realities more meaningful in its cultural context. He proposes that western art, in general, is connected in purpose when discussed in the surrounding historical events and motives. Western artists, starting from panoramas ending with virtual reality experiences, have been trying to bring a virtual experience or what he refers to as virtual (experiential) art that takes the audience beyond space and time to a new reality. Similarly, this is the essence of extended realities in its three forms: virtual, augmented and mixed. These attempts started by creating illusions (optical, auditory and tactile) that got more immersive as techniques and technology developed (Grau, 2003). 


\subsubsection{What Constitutes Extended Realities}

The body is the medium to build a world around us; this has been done throughout history by alternating our existence through the utilization of different instruments (Merieau-Ponty, 1962). In other words, we are able to live in a symbolic reality that constitutes a new dimension, and through this capacity we produce an existence (Carey, 1989). In the virtual realm, what really makes a difference is the art of immersion: how to immerse the body into that form of storytelling to create that virtual world. Reflecting on these points of view in the context of extended realities, these platforms are based on the experiential elements of storytelling which could be seen as a true representation of how life is experienced and what gives realism theory its new facelift. As a result, it grants the significance of this shift from conventional storytelling towards an experiential one.

\subsubsection{Affordances}

Extended Realities offers many affordances but for the reasons of this research we will focus on few main ones. These are:

1. Immersion

2. Interactivity

3. Embodiment / enactment

\subsubsection{Immersion in extended realities}

Murray (1998) describes immersion as "the active creation of belief", in contrast with Coleridge's description of "the willingness of suspension of disbelief" (Sadowski \& Stanney, 2002). Baudrillard (1995) describes it as an agreement between the stakeholders that they will believe and experience what is not real and assume its realism. Psychologically, Singer and Witmer (1999) describe it as the psychological state in which the participant is interacting with an enveloping environment which as a 
result provides an experience. Additionally, Singer and Witmer (1999) describe it focusing on the technology itself while the majority of researchers lean towards the psychological aspect.

Types of immersion

Immersion is, arguably, the most important factor in creating a realistic experience and true presence. It is one factor that does affect all other factors in an intersected way, therefore, there have been so many research studies trying to figure out the different types of immersion and compare them. The main types of immersion could be classified as follows:

\subsection{Strategic Immersion}

In strategic immersion, the participant is given a situation in the future to plan with alterations of the present experience. This focus on the result rather than the present action makes the participant awareness of the present more forgiving (Murray, 1998; Isbister, 2017).

\subsection{Tactical Immersion}

This type is based on the stimulation of the tactile sensory; the immersive experience would focus on high-level skill-set or repetitive simple actions. This is one of the most common approaches in game design (Papagiannis, 2017; Isbister, 2017).

\subsection{Spatial Immersion}

This type focuses on the quality of the virtual space created around the participant. It is directly related to the fidelity level when creating the environment. The design is perfected as much as possible to avoid distracting the participant or disengaging them due to low resolution or quality. This highlights the importance of the technology involved and its level of fidelity (Slater \& Wilbur, 1997; Isbister, 2017). 


\subsection{Narrative Immersion}

Although this is the most common amongst all, it is the most difficult to perfect. It should be a nice mix of interesting storylines that is flexible to maintain the value of the participant's choices. The main challenge is the difficulty in setting different meaningful outcomes for the same storyline. However, this interactivity is one of the most important affordances in extended realities (Murray, 1998; Papagiannis, 2017).

\subsection{Social Immersion}

One of the major strengths of augmented reality (AR) is that it produces higher feelings of social immersion (Billinghurst \& Thomas, 2011; Papagiannis, 2017). The social context of the experiences is another immersive enhancing element; "Pokemon Go" (Pokemon Go, 2018) and Tilt Brush (Tilt Brush, 2018) are great examples. TiltBrush, in particular, is an AR drawing application that could be described as the tool the transforms that world into a canvas. It gives the opportunity to do things that are not socially acceptable in real life, such as drawing on walls.

This social interaction, known as social play, combines competition, cooperation, and coopetition in an emotional context. However, the physical challenges are what make it interesting; if the experience offers no challenge it would never move the players emotionally (Isbister, 2017).

\subsection{Context Immersion VS Sensory Immersion}

Kim (2013) differentiates between sensory and context immersions in Mixed reality experiences, specifically. They focus on mobility as a big element in their debate. 


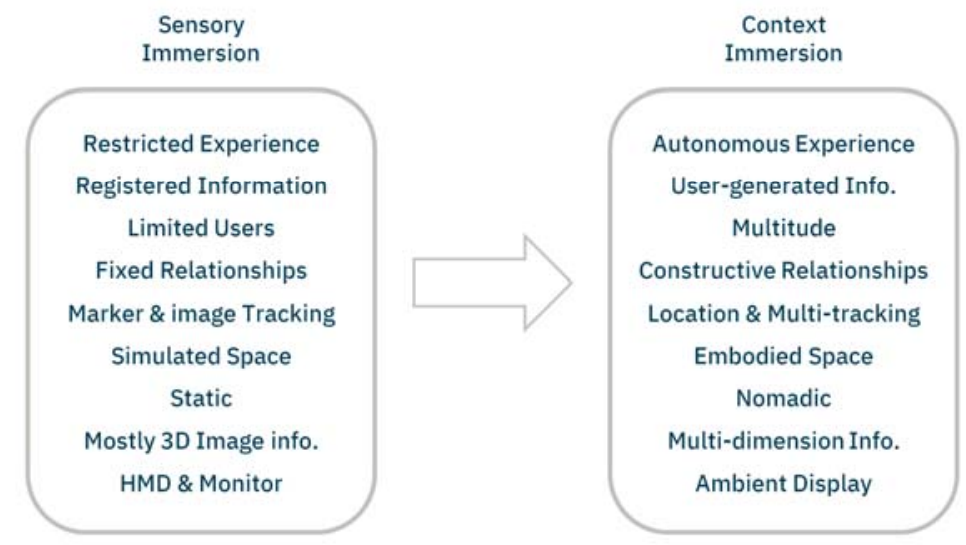

Figure 1. The characteristics of the context immersion (Kim, 2013)

They propose three dimensions of the context immersion in mixed reality environments for Mobile applications. The dimensions are time and location-based context, the object-based context, and the userbased context immersions. These three integrate and affect each other based on the figure below (Kim, 2013).

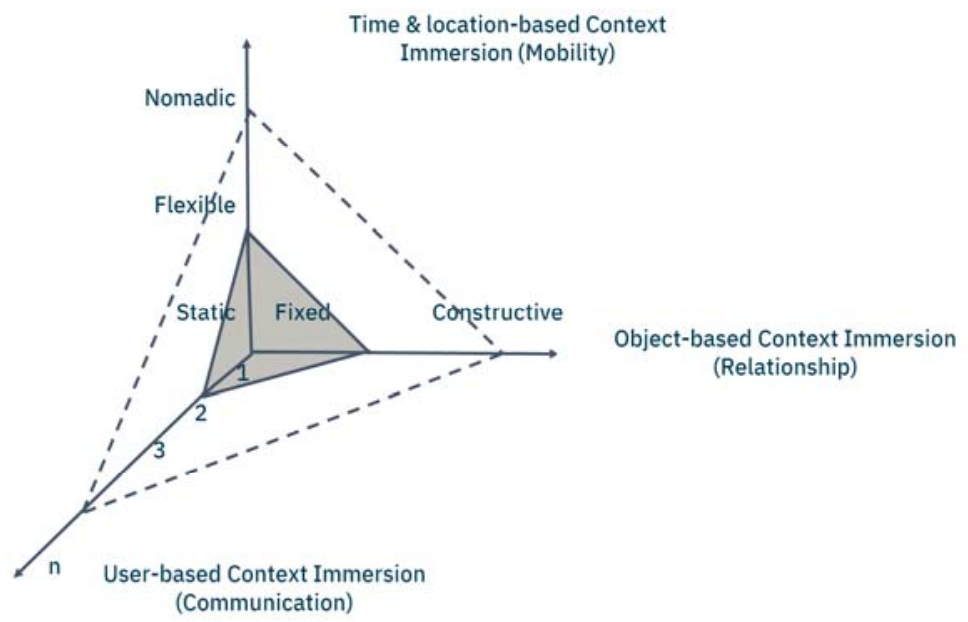

Figure 2. Dimensions of the context immersion in the MR environments (Kim, 2013)

They state that context immersion is based on the user personalization and interactivity which makes it more immersive and fulfilling for the user (Kim, 2013). 


\subsection{Emotional Immersion}

The emotional immersion is, arguably, one of the strongest as it is mainly based on the brain's ability to see things in a certain way. Therefore, Isbister (2017) focuses in her work on design for emotions based on game design. Game designers have been very effective in tackling this type of immersion. The more researchers understand the way the human brain works, the more they will be able to tailor the technology to enhance the extended realities experiences. One of the most promising projects in this regard is the Blue Brain Project in Switzerland, which is attempting to reverse engineer human brain to study it more in-depth (Blue Brain, 2018).

\subsection{Hybrid Immersion}

It is worth mentioning, that in practice, immersion is not is not that abstract or simple. It is a hybrid form or mix of immersions. This mix is dictated by the nature of the experience and the foreseen outcomes.

\subsubsection{Interactivity and nonlinear storytelling}

There are a lot of studies about the role of interactive fiction for different platforms. Interactivity gives the audience the opportunity to make meaningful choices that could alter the story being told (Cavazza, Charles, \& Mead, 2002); this is an element that increases immersion as discussed earlier. Additionally, it facilitates individualized non-linear form of storytelling. It could be argued that this is the main element that differentiates true virtual reality films from 360 films.

\subsubsection{Embodiment/ Enactment}

\subsection{Embodied Wearable Technologies}

Although it is not as distinctive as the psychological aspect of immersion, Embodiment has to be considered within its technological context (Heeter, 1992). A traditional example of embodiment is getting used to prescription lenses to such a level that they are not noticed anymore. Wearable 
technologies are based on the same concept. Pearlman (2015) categorizes cyborgs into three types: those that use mechanical parts, those that use electronic parts and those that use the cybernetics; however, a cyborg could belong to two types at the same time. When technology embodiment is truly achieved immersion is complete.

Although the literature proposes that extended realities are disembodiment media, it is misleading given the crucial role movement and body plays in extended realities nowadays (Kuppers, 2003).

The perfect immersive experience is an experience that would make the participants forget that they are wearing headsets or that their movement is limited spatially. These experiences give participants the chance to get rid of any constraints that interrupts their experience. It gives a higher level of movement freedom that allows multi-player experiences and social interactions. Therefore, big companies are now introducing wireless headsets such as HTC Vive wireless adapter (Vive, 2018) to provide a stronger sense of immersion

\subsection{Performer VS Viewer}

The audience is no longer a group of viewers; they are participants/ cyborgs, which would allow them to become performers. It is one of the main reasons behind the appeal of the medium in the context of the participatory culture we live in (Delwiche \& Henderson, 2012).

Similarly, method acting relies heavily on immersion as actors trick their minds to believe that certain circumstances are real. Or what the famous acting coach Sanford Meisner refers to as imaginary circumstances (Meisner, Longwell, \& Pollack, 1987). It is possible that using method acting techniques in emotional triggers could help creatively in building an increased emotional immersion. This would give the opportunity to reflect on the golden box and emotional triggers theories in method acting, which are based on the fact that all human beings have a storage of all types emotional reactions and all it takes is creating proper emotional triggers to evoke these emotions (Moore \& Logan, 1984; Meisner, Longwell, \& Pollack, 1987; Adler, 1988; Chekhov, 1993; Barr, 1997; Kazan, 1997; Moss, 2005; Hagen, Pierce, \& 
Frankel, 2009). However, this escalated immersion could pose the risk of losing control. There have been many speculations stating that many sad incidents such as the death of Heath Ledger, were caused by the over-commitment to the practice of method acting (O'Brian, 2013); although this cannot be verified without proper clinical research, it is a risk that should be considered.

\section{Fidelity}

Fidelity in the context of extended realities refers to the authentic representation of a simulation. The higher the fidelity the more authentic is that representations and vice versa. The importance of fidelity lies in its relation to immersion and presence; the higher the fidelity, the higher the immersion and presence. Spetial fidelity model of presence is based on the concept that presence is dependence on the degree of similarity of spatial, auditory, and haptic transformations between virtual and real realities (Barfield \& Hendrix 1995, in Bystrom, Barfield, \& Hendrix, 1999; Barfield, Hendrix, \& Bystrom 1997 , in Bystrom, Barfield, \& Hendrix, 1999). This fidelity is affected by many factors such as geometric field of view, dieplay update rate (Barfield \& Hendrix 1995 , in Bystrom, Barfield, \& Hendrix, 1999). On the other hand, Bystrom et al. (1999) refers to it as the sensory fidelity in the integrated immersion, presence and performance model, which is displayed in the following diagram. Understanding this concept highlights the importance of technological advancement in building simulations and experiences. 


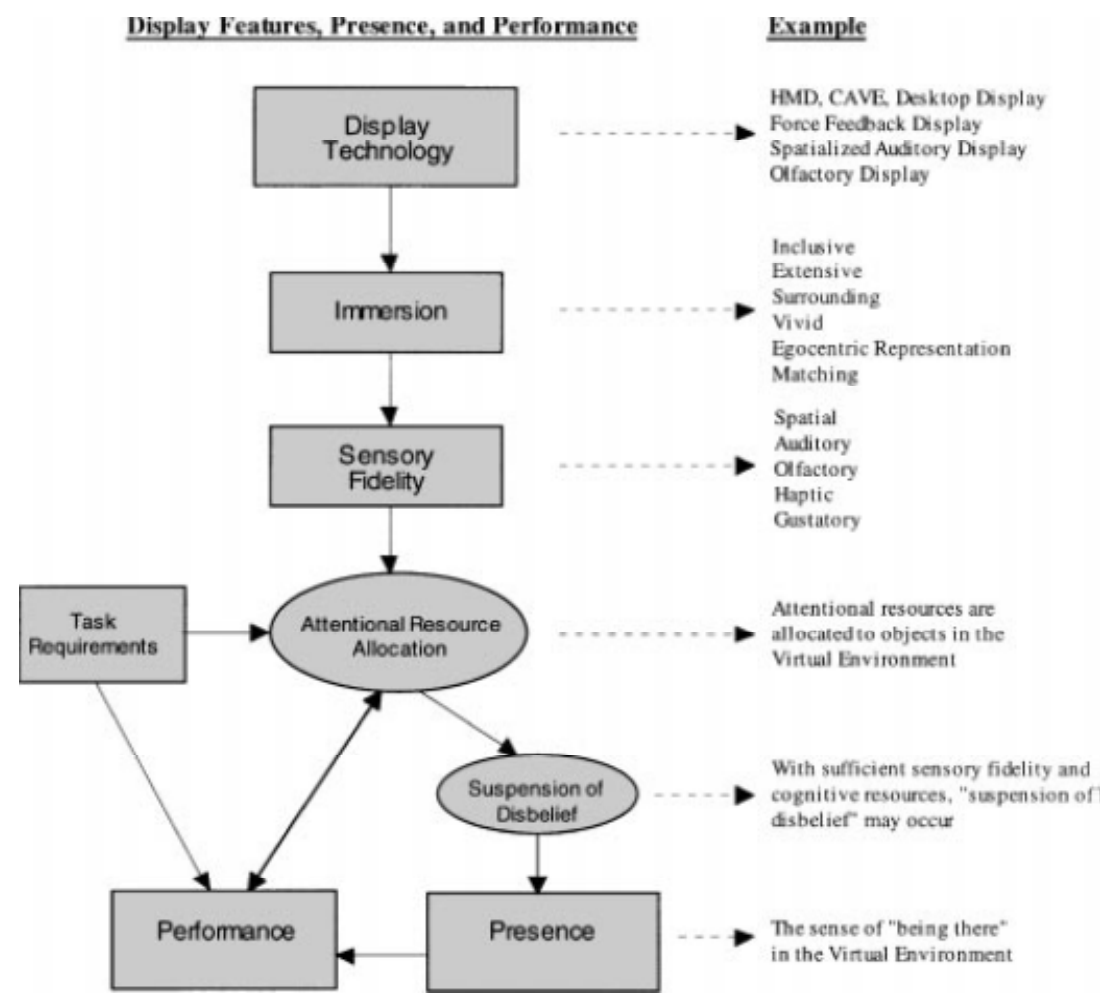

Figure 3. Diagram of a model on immersion, presence, and performance (Bystrom, Barfield, \& Hendrix, 1999)

\subsubsection{Presence}

Presence is the state of being present in the moment; it is governed by physical (external) factors (Barfield \& Hendrix, 1995; Slater \& Wilbur, 1997) and psychological (internal) factors (Heeter, 1992; Singer \& Witmer, 1999), while Heeter (1992) categorizes presence into three different types: environmental, social and personal.

\section{Design for emotions}

\subsubsection{Game design and user experience}

There are certain elements in games which differentiate them from other types of media; these elements are what make games emotionally immersive. For example: meaningful choices, entering the flow state 
which is a pleasurable optimal performance, social emotions, social play, and movement design. (Isbister, 2017).

The Importance of Social Play

Human beings by nature flourish through social interactions. There is something fulfilling about bonding over obstacles and coordinating activities to reach a certain outcome. Participants in games agree to experience a social interaction with alternative identities in virtual circumstances through role-playing to achieve a common shared goal. In the broader context, Walter Mischel pointed out the power that social situations have on the way individuals portray themselves (Mischel 2004, in Isbester, 2017); this is especially significant when participants express themselves through avatars. Social play allows players to bond over obstacles, and encourage them to coordinate activities to reach a shared desired outcome.

\subsubsection{Social Emotions and Transformation}

Games trigger "social emotions", which are the emotions people experience in their relationship with others in a social context: as players get immersed, they start feeling a social connection towards the characters in the game as if they were real. According experts in grounded cognition, this is achieved through evoking emotions by mirroring the way human brains comprehend the world around us. This field explains how games affect participants while the players are taking alternative identities (or avatars) or social situations (Isbister, 2017).

Murray (1998) highlights that "transformation" is one of the main aesthetics of the medium; it is the ability to change into fancy characters or transform things around the users into different shapes or characters; it is the ability to have transformations and role-plays that are impossible in real life.

\subsubsection{Meaningful Choices}

These choices are what makes the players feel their value in the game, they can change the outcome of the story; they become proactive instead of passive (Isbister, 2017). Murray (1998) calls it the "agency"; it is 
the powerful beauty of taking action and seeing its positive outcome. The more immersive the experience, the stronger the sense of agency is. Participants like to be active in these environments. There is a special sense of pleasure in solving problems proactively instead of witnessing them unfold passively (Murray, 1998). Researchers have found that games activate the "Reward-Related Mesolimbic Neural Circuits" more than watching TV, while shifting the emotional patterns observed in the participants' brains (Isbister, 2017).

\subsubsection{Flow}
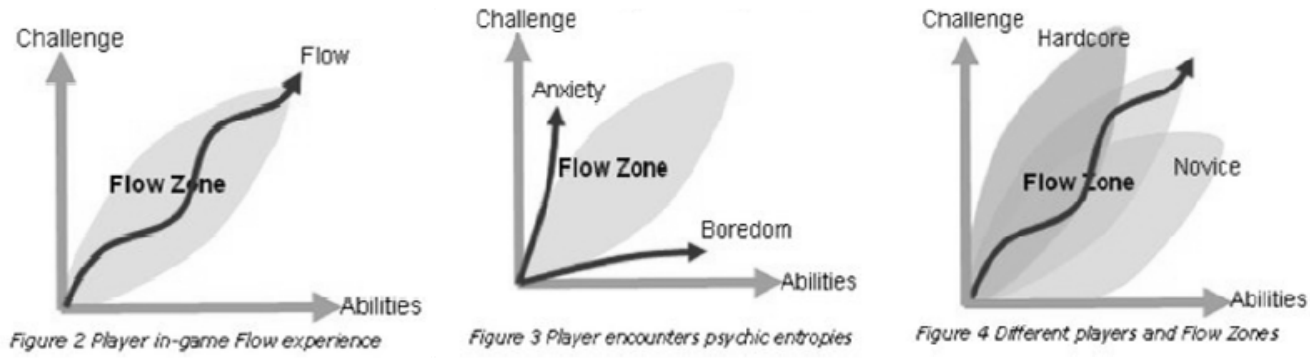

Figure 4. Different Players and flow zones (Chen 2006 in Isbister, 2017)

An important element is the ease through which a player can enter a pleasurable optimal performance state; this state is called the "Flow" state as referred to by psychologist researcher Mihaly Csikszentmihalyi (Isbister, 2017).

\subsubsection{Movement Design}

Using movement design to create emotion and connection is another big element, research has proven that using certain movement would affect human emotions. Body language experts have found that by changing our posture we would change our mood, social psychologist Amy Cuddy talked in a TED talk about the effect of striking high power pose for couple of minutes and how it would make individuals feel more powerful in contrast to going for low power pose (Isbister, 2017). 
Isbister (2017) states that emotional cognition is based on body movement and offers two immersion advantages to game designers, because participants who witness the movement would be affected to some extent by it, while virtual characters (the living others, non-players) could be used to evoke emotions in participants.

\subsubsection{Word of caution}

Emotional immersion could be a double-edged sword. One of the most significant indicators of how powerful is emotional immersion in game design is video game rage. It is a result of an uncontrollable emotional immersion that showcases how dangerous it could be if it was not handled properly or used negatively. A recent example is the "Blue Whale" social game (Forbes, 2018).

\subsection{Design for empathy}

Digital Media carries social and ethical responsibilities and utilizing these theories in the context of empathy machines would bring future cinema one step ahead in the right direction.

\subsubsection{Empathy}

Despite the rich psychotherapy literature on empathy, researchers don't agree on a unified definition yet (Bohart \& Greenberg, 1997; Batson, 2009). One of the main turning points in identifying empathy processes in the brain was the discovery of mirror neurons in the motor cortex of macaque monkeys and the introduction of action mirroring theories (Gallese, 1998); However, Human empathy seems to be more complicated in nature; and according to Elliott et al. (2011) there is an increasing evidence that empathy in the human brain consists of three major neuroanatomically based sub processes: an emotional simulation process (Decety \& Lamm, 2009), an emotion-regulation process (Decety \& Lamm, 2009), and a conceptual, perspective-taking process (Shamay-Tsoory, 2009).

Rumble et al. (2009) instead argues that empathy is influenced by two conditions: Perceiving individuals who are struggling; and Imagining their emotions, circumstances and struggle. Meeting these conditions 
would increase empathy; and in turn, most likely, increase positive (generous) behavior (Batson, 1991; Batson et al., 1996; Batson, Early, \& Salvarani. 1997).

\subsubsection{Awareness VS empathy}

It is important to differentiate between awareness and empathy. Reflecting on the definition it is obvious, that awareness is based on knowledge while empathy is based on emotions and experiences. Based on this observation, it is important to design for emotions and empathy together in an integrated way.

The rise of empathy machines

Research has shown that virtual reality (VR) Platforms have facilitated an increase in empathy when used for news storytelling (Archer \& Finger, 2018). Furthermore, Chris Milk (2015) proposes VR as the ultimate Empathy Machine. Empathy Machine refers to a technological and content-based approach to represent someone else's emotional experience, with the goal of inhabiting another body. In this context, virtual reality content attempts to embody someone else's experiences as a more successful means of creating empathy when compared to more traditional media forms. This embodied experience, it is suggested, is more successful at stimulating the empathy circuits in the human brain (Bollmer, 2017).

Milk's (2015) bold claim wasn't received lightly. For example, Bollmer (2017) proposes using radical compassion instead of empathy, stating that empathy is usually done in a meaningless way, and is difficult to tackle. However, this argument is based on empathy machines in VR context. As this paper explores, augmented reality (AR) and mixed reality (MR) platforms offer affordances that may be more suited to empathy machine experiences. These affordances could increase the immersive nature of the user's action and, hypothetically, could increase empathy. This, as a result, could increase the meaningfulness of empathy machines and address the issues stated in Bollmer's argument. 


\section{Alzheimer's disease}

In the search for a theme for building empathy experiences, it is important to pick a particular domain that embodies a social need. This process went through different stages while choosing different themes. Reflecting on a personal experience while filming "Silence", which is an experimental short film that tells the story of an Alzheimer's patient, I realized that there is a huge misunderstanding of Alzheimer's disease. It was obvious that this theme, potentially, could serve a stressing need for a better understanding of the disease, in general, and patients' needs, in specific.

International AD organizations (Alzheimer's Society, 2018; The Alzheimer Society of Canada, 2018; The Alzheimer's Association, 2018) describe Alzheimer's progression in an intersected fashion, with each stage attacking a new region in the brain. It is difficult to pinpoint every single change that affects the brain in this gradual progression. However, for the reasons of this project, and in order to simplify the learning process, we will attempt to categorize this progression into 8 progressive stages based on the main affected regions at each stage, as follows:

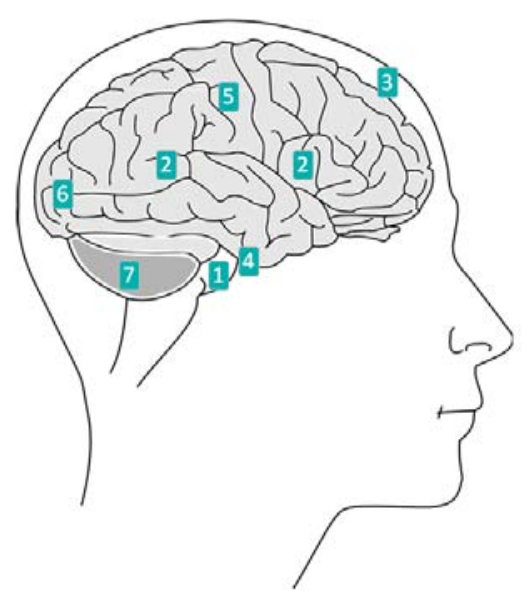

Figure 5. Alzheimer's progression simplified: (1) Hippocampus (2) Wernicke's and Broca's areas (3) The cerebral cortex (4) Amygdala (5) The Sensory Cortex (6) The back of the brain (7) The cerebellum. 
1. Hippocampus: where memories are formed, once damaged it becomes gradually harder to form new memories.

2. Wernicke's and Broca's areas: where language is processed, once damaged it becomes more difficult to find the right word.

3. The cerebral cortex: where the logical thoughts, solving problems, grasping concepts and making plans and solving mathematical equations happen.

4. Amygdala: where emotions are regulated, and once damaged the patient loses control over their mood and feelings.

5. The Sensory Cortex: where the brain makes sense of what it sees, hears and smells; the damage to this area can spark hallucinations.

6. The back of the brain: where the most important and emotionally high-impact memories are stored.

7. The cerebellum: This region functions to coordinate voluntary movement that compromises balance and coordination.

8. The last stage destroys the part that regulates breathing and the heart, which causes death.

For obvious reasons, stages 6 and 8 are omitted from the experience. We will focus on six major stages for the sake of our simulation and experience design.

\subsection{Aging symptoms}

One of the major factors that contribute to the severity of Alzheimer's disease is that it is accompanied by symptoms of aging, including:

\subsubsection{Visual aging symptoms}




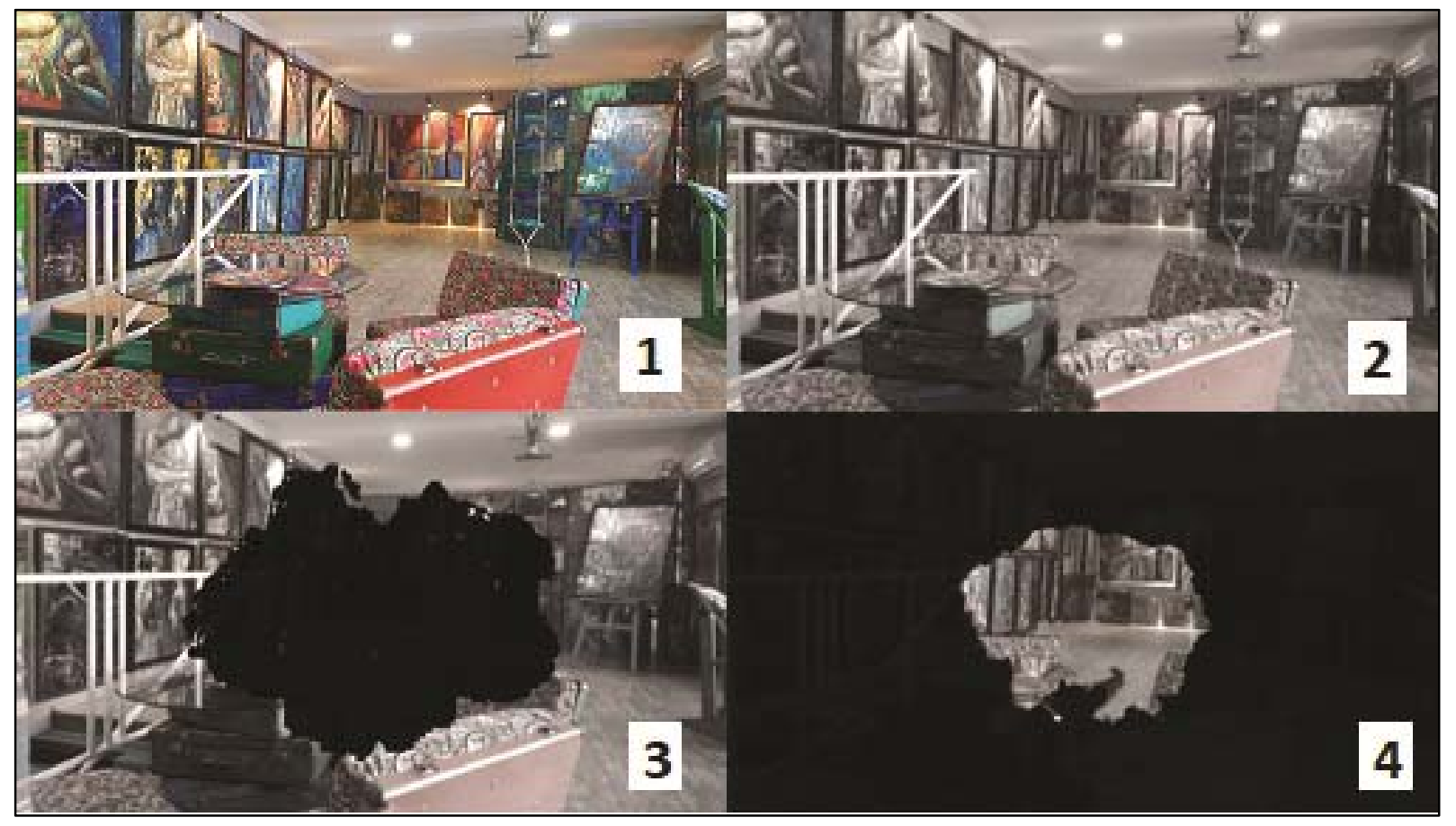

Figure 6. Visual aging symptoms: (1) Normal (2) Cataract, (3) Macular Degeneration and (4) Glaucoma.

\subsubsection{Cataract:}

Cataract is a clouding of the eye lens which leads to a decrease in vision. Cataracts often develop slowly and can affect one or both eyes. It is characterized by the following:

o Foggy or cloudy vision.

o Blurry vision.

o Problems with glare.

o Dull color perception.

o Increased nearsightedness.

o Frequent prescription changes.

\subsubsection{Macular Degeneration:}

This phenomenon is perceived as a large spot in the center of one's field of vision. 


\subsubsection{Glaucoma}

This condition is commonly referred to as tunnel vision. In some cases, the patient sees a sharp center with blurry corners.

\subsubsection{Auditory aging symptoms:}

Samples include:

o Hearing impairment and reduction in fidelity.

o Tinnitus: it is a condition that causes a continuous sense of ringing in the ears.

o Noise: It has been reported that some seniors experience noises and radio-like distortion.

\subsection{Gamified Education}

\subsubsection{Problem based learning}

It could be argued that an education that doesn't relate to the workplace and doesn't equip students with the skills required for employment is incomplete. It is the teacher's responsibility to develop life and mental skills that would help students in their progress both academically and career-wise, is incomplete. This type of education is referred to as the "Problem Based Learning" or "Hands on Learning". It was found that this type of learning increases students' engagement and excitement in information literacy instruction (Glusker, 2017). 


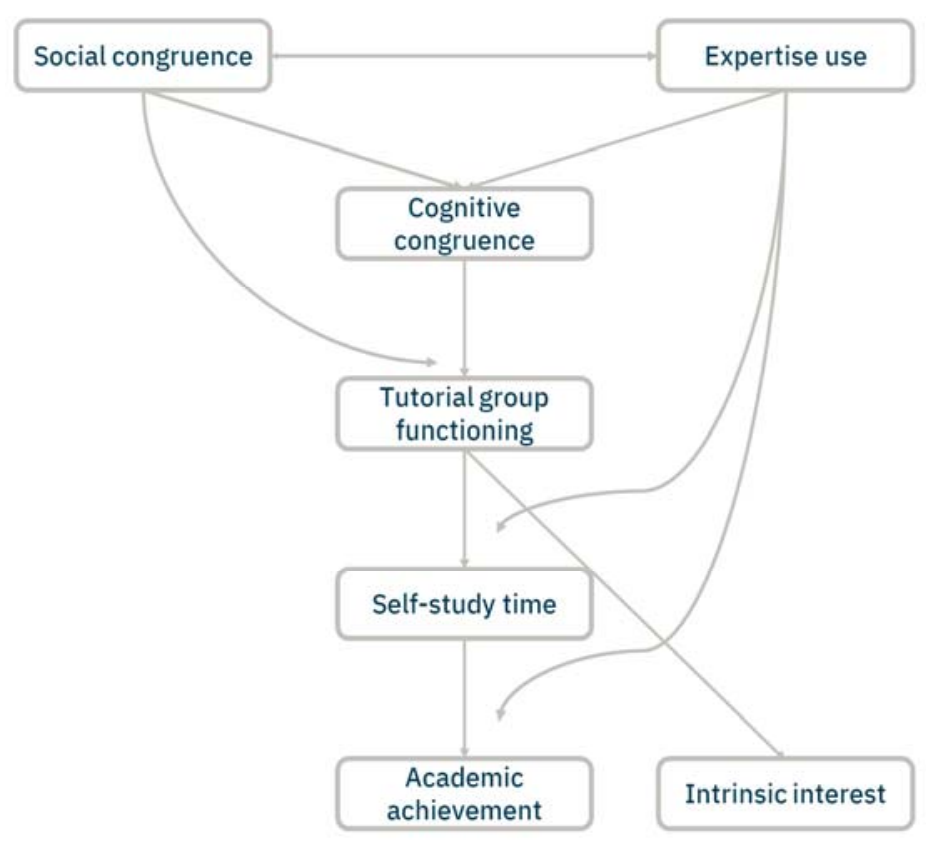

Figure 7. Adapted from the less restrictive variant of the effective-tutor model (Glusker, 2017) However, such approach can't be proposed just as an idea. Therefore; academics have been developing flowcharts to standardize and guide the teachers through their implementation of this learning approach. This research has led to many approaches including gamified education and serious games.

\subsubsection{Serious games}

Serious games is a term used to describe games that educate, train and inform (Michael \& Chen, 2006). The approach adds elements that, we propose, could further add to the empathetic effect to the experience. Serious games design should balance the challenges and participants abilities, which would be achieved through the mechanism proposed in figures 8 and 9 (Westera, 2016). 


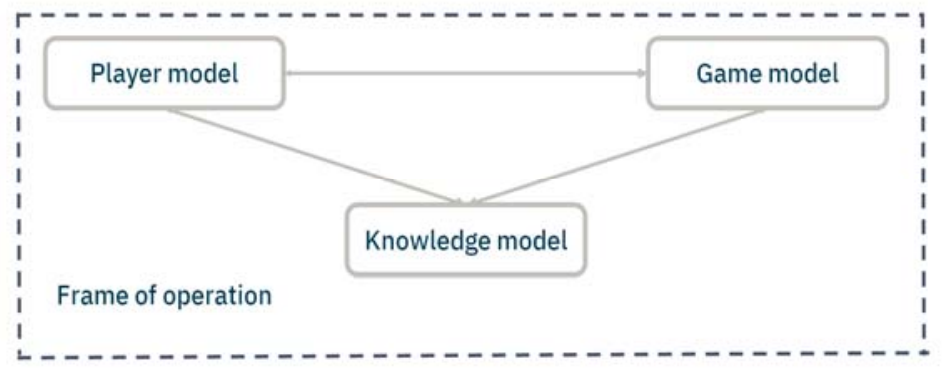

Figure 8. Serious gaming model components. (Westera, 2016)

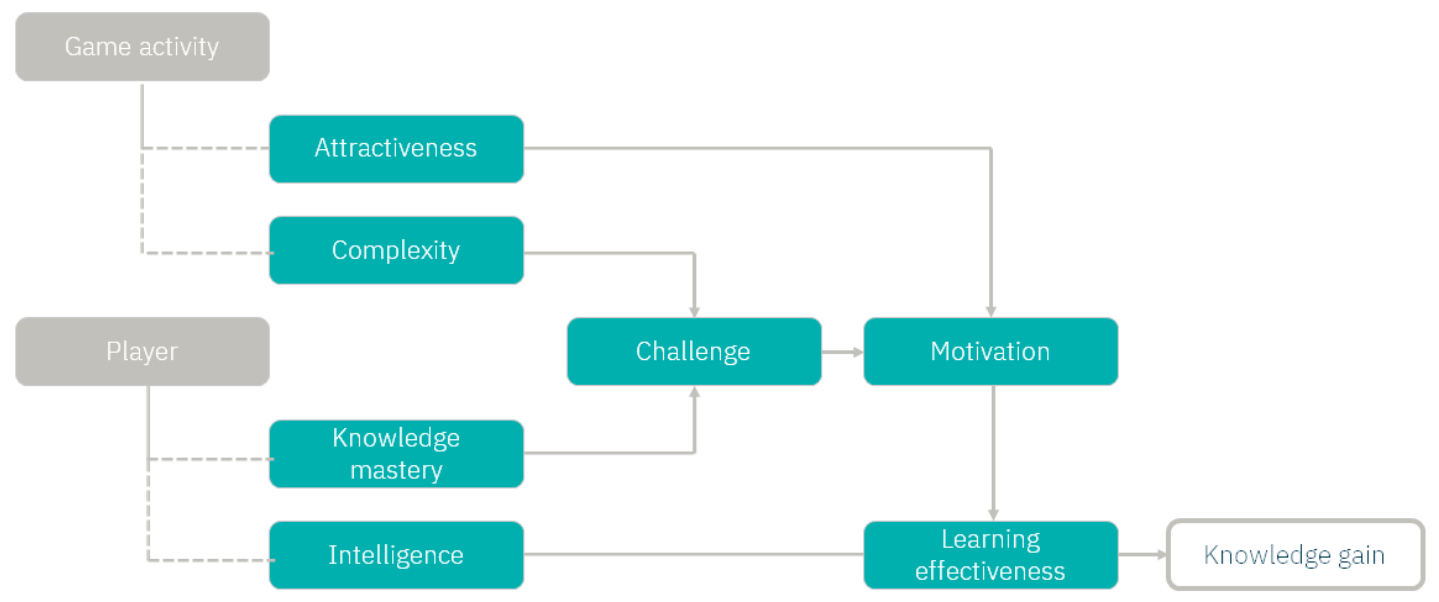

Figure 9. Adapted from the causal model of learning upon engaging in game activity (Westera, 2016).

Researchers have suggested that specific serious games interventions show signs of increasing personal and social learning and ethics as shown in figure (3) (Pereiraa \& els., 2012).

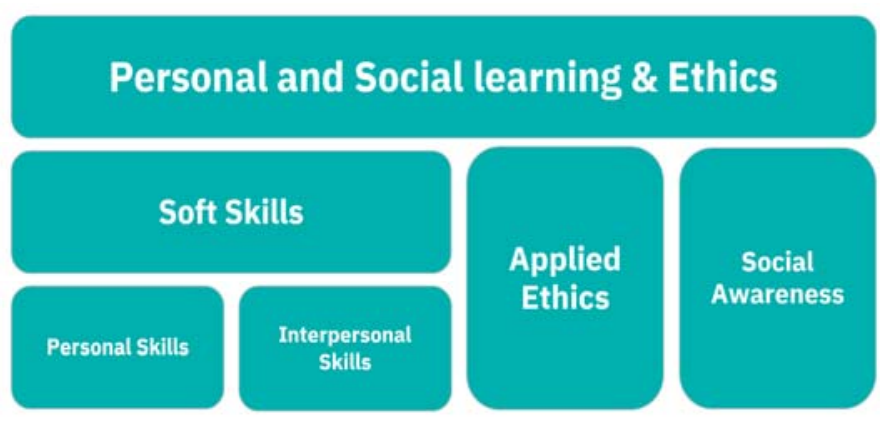

Figure 10. Taxonomy higher-level (low-detail) categories (Pereiraa \& els., 2012). 


\section{The methodology}

Reflecting on the above mentioned factors, it is obvious that augmented and mixed realities could be great platforms for creating empathy machines. However, virtual reality is less effective because of the idea that the embodiment is not as efficient and as complete as it is when it comes to other extended realities. This, as a result, affects the emotional immersion.

Moreover, interactivity and design for emotions are two major contributing parts and are appreciated by the participants because they transform them from viewers to performers. This is a true manifestation of the participatory culture that has been developing over the years (Delwiche \& Henderson, 2012).

In order to really empathize with others, participants have to experience other's realities. It is the next level of empathy that could be experienced through the technological development. Empathy by definition is the state of understanding other people experience and no one could understand someone else's experience truly unless they live it themselves. And in this time and age participants are capable of experiencing them by replicating these experiences to a very high extent psychologically (emotionally) and physically. The more researchers understand how the brain works the more it will be possible to manipulate it. To date, it could be argued that humankind is at the peak of developing empathy machines. And to utilize all of the previously mentioned factors, the following chart was developed as the basis for the immersive design for emotions and empathy in extended realities. 


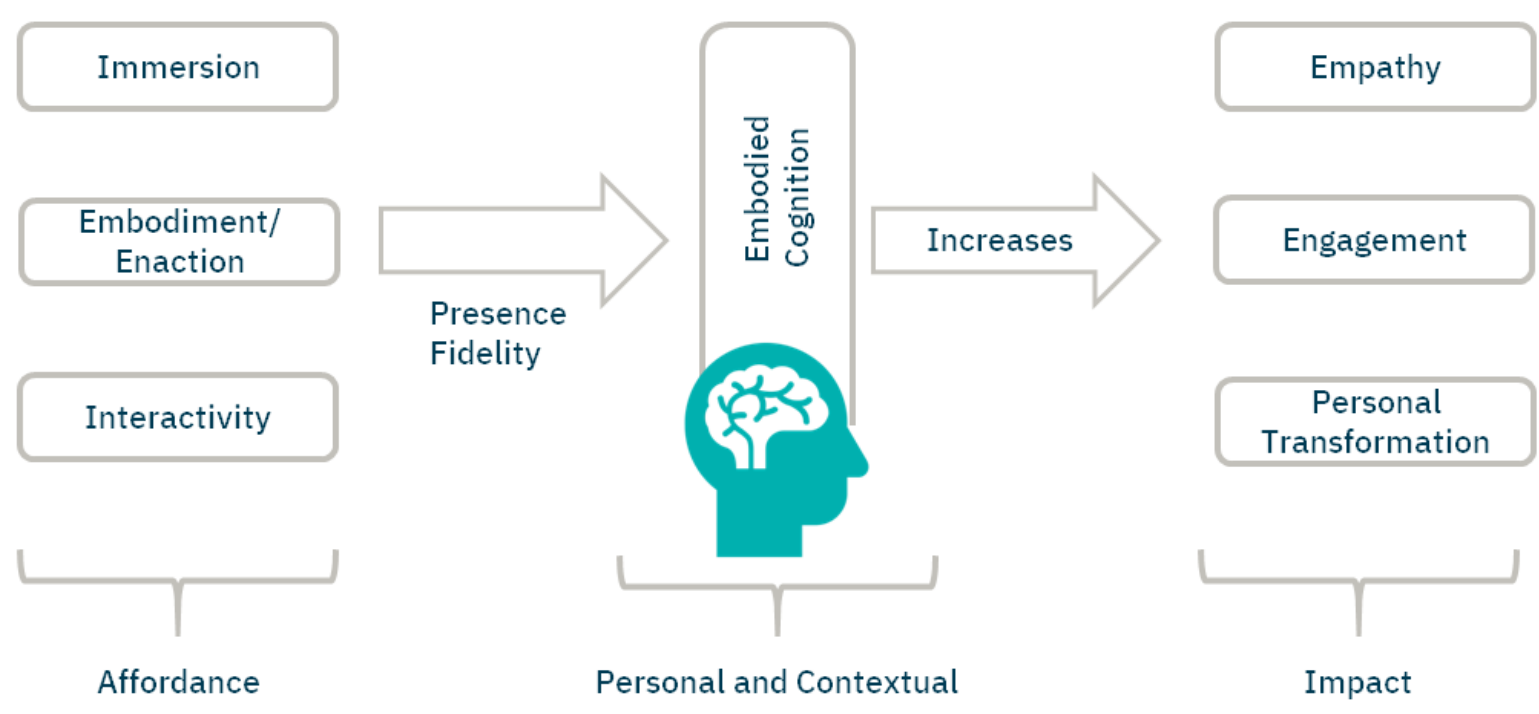

Figure 11. immersive design for emotions and empathy in extended realities.

Isbister's design for emotions (2017) shows that certain elements in game design, for our purposes, differentiate them from other types of media. To increase impact and engagement, we are including game-like properties in our experience design, such as:

o Meaningful user choices.

o Entering the flow state.

o Social emotions and play.

o Movement Design.

These experiences are designed to deliver a highly individualized form of story experience achieved using a thorough understanding of design for emotions and empathy techniques (Isbister, 2017), the medium interactivity, the impact of non-linear structured experiences, and subconscious mind's influence on human behavior based on Freud's work (Freud, 1899). However, each experience is not necessarily telling a story but rather delivering an experience. Since this approach is an attempt to copy life experiences, it is not necessary that every experience tells a story on its own, but as an accumulation, it 
could tell a story around a certain theme (in this case, focused on Alzheimer's). Some examples that come to mind are dreams, nightmares, and memories. They don't necessarily tell a story on their own but they are experiences that would construct a story if blended together. Carey (1989) states that reality is a result of constructing, apprehending and utilizing symbolic forms.

The whole experience design in this model is approached in a way that simulates life; experiences have different impact on us based on their sequence of occurrence. This model is meant to make these experiences part of their life experiences that last longer than commercial transmedia storytelling. In other words, it is an attempt to make the film's influence more concrete in individuals' subconscious. What makes augmented reality experiences more viral is when it becomes invisible because of its ease to learn and use, while the participant is the controller of everything (Papagiannis, 2017).

Virtual and mixed realities are two main platforms that are utilized in this model because their affordances are a perfect match to the required outcome. One main affordance in virtual reality is experiencing the story in 360 degrees, conveying dreams, nightmares, and memories with a realistic immersive feel (Dachis, 2016). Moreover, both platforms allow using realistic sound design based on binaural sound technologies. On the other hand, mixed reality has the ability to bring the story into what seems like a physical location; this influences the audience to live the experience rather than witness it or interact with it.

While using these three platforms, issues of the likes of the suitability of the medium for the storytelling style will be addressed. It is argued that virtual and mixed realities are not mediums of storytelling but rather mediums of experience; one of the aims is to embrace their true strength in being experiential platforms. In this project, the attempt is to build a believable story that would contribute to the audience transformation in an impactful way. The audience is going to do the rest of the job. 


\section{Hypothesis}

Empathy by definition is the state of understanding other people. Our project attempts to build empathy through immersive design and serious game dynamics in extended realities. Our hypothesis is the gamification of empathy machines will achieve better results than other methodologies.

\section{Results (Designed Extended Reality Experiences)}

Building on the methodology mentioned earlier, two experiences were designed; "Silenced Memories" which is an interactive virtual reality experience and "Alzheiemr's Eyes Challenge" which is a gamified mixed reality experience.

These experiences were created as elements in a transmedia storytelling campaign inspired by an experimental short film called "Silence" about an Alzheimer's patient.

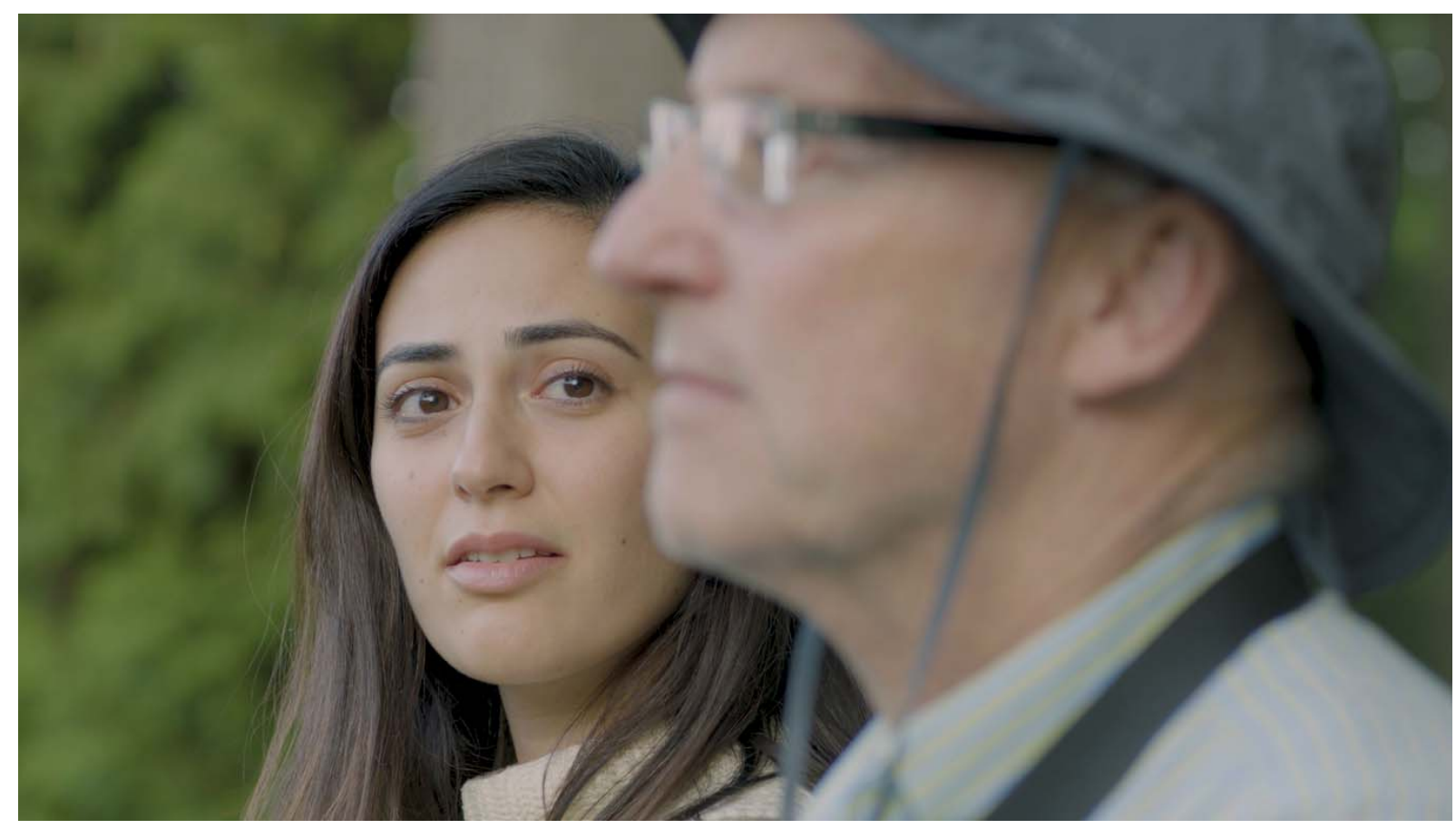

Figure 12. A shot from "Silence", a short film by Assem Kroma, to which this experience is complementary. 


\subsection{Silenced Memories}

\subsubsection{The idea}

An interactive non-linear experience based on the fact that doctors measure dementia progression through patients' ability to draw.

It is a non-linear form of storytelling. The participant experiences memories and as a conclusion, they start shaping an understanding of who they are. They are surrounded by darkness; they can't see anything, the memories will haunt them one thought after another. They see each memory then it blacks out. Memories are a mix of black and white, colored, still images, video and in some cases just a sound. They are a mix of different life experiences.

\subsubsection{The storyline}

The participant is Alzheimer's patient, they are experiencing memories, and they don't necessarily understand that they are suffering from Alzheimer's disease.

\subsubsection{The elements}

This part of the project was designed around four main thematic elements of human life:

1. Temporariness: faith and death.

2. Innocence and unconditional love: childhood memories and mother-child relationship.

3. Sensuality: love and dance.

4. Human vulnerability: depression.

\subsubsection{Technical design}

This component was designed to be experienced in HTC Vive, and the user experience is as follows:

1. The intro starts playing and the thematic images appear one after another.

2. User looks at a photo

3. A photo timeline is triggered, the main screen disappears 
4. The timeline for that theme starts playing.

5. After the timeline is finished, a memory-related noise starts playing in the background.

6. The main screen appears again, the following changes are incorporated:

a. The photo, that has been already triggered, disappears.

b. The noise related to that memory starts playing until the end of the experience, in an accumulative manner.

7. The rest of the photos appear and are interactive, (i.e. the participant could trigger new events by looking at them).

8. Repeat

a. More photos appear: repeat

b. All photos have disappeared: all memory related noises have been triggered and the participant is left with a unique individualized emotional state.

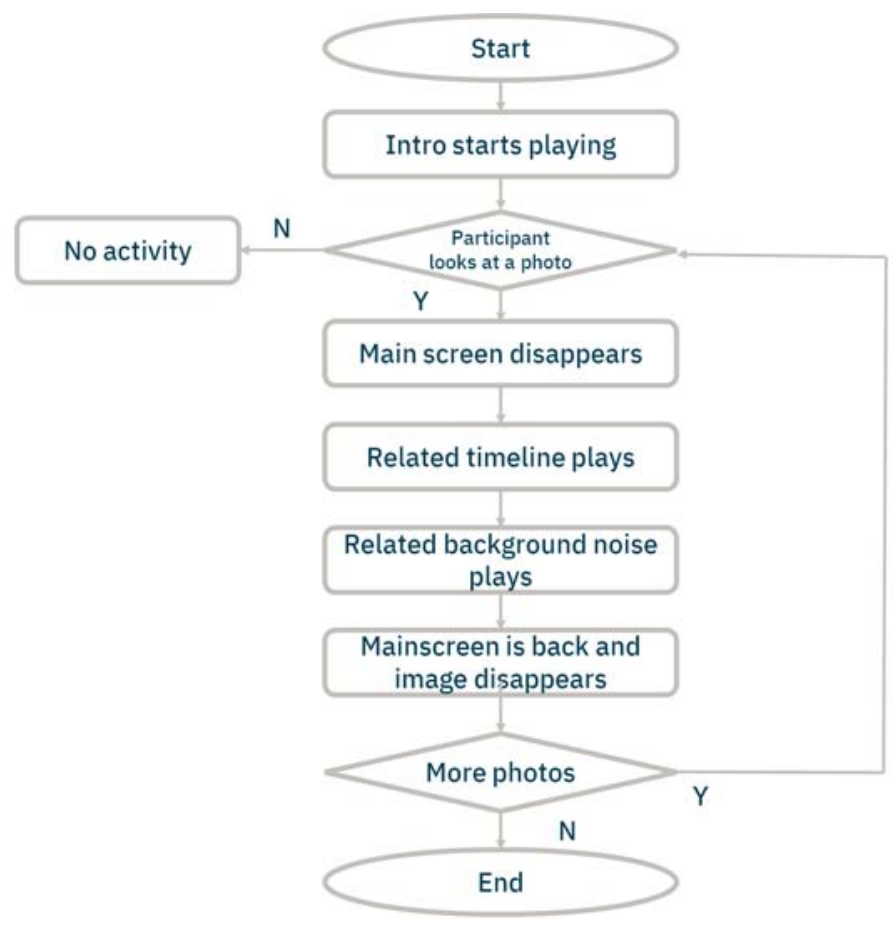

Figure 13. Silenced Memories' User experience flow chart. 


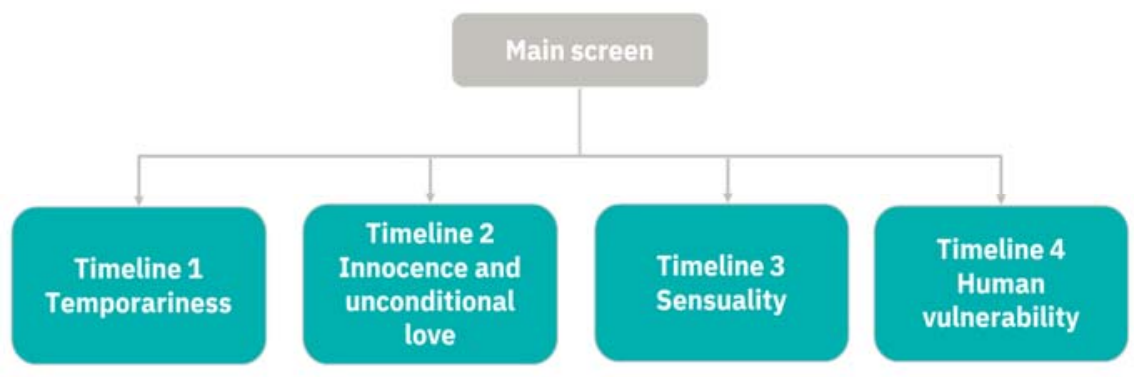

Figure 14. The experience technical build hierarchy.

The experience is interactive based on head movement tracking; as a participant, I wear the headset to find that I am surrounded by darkness.

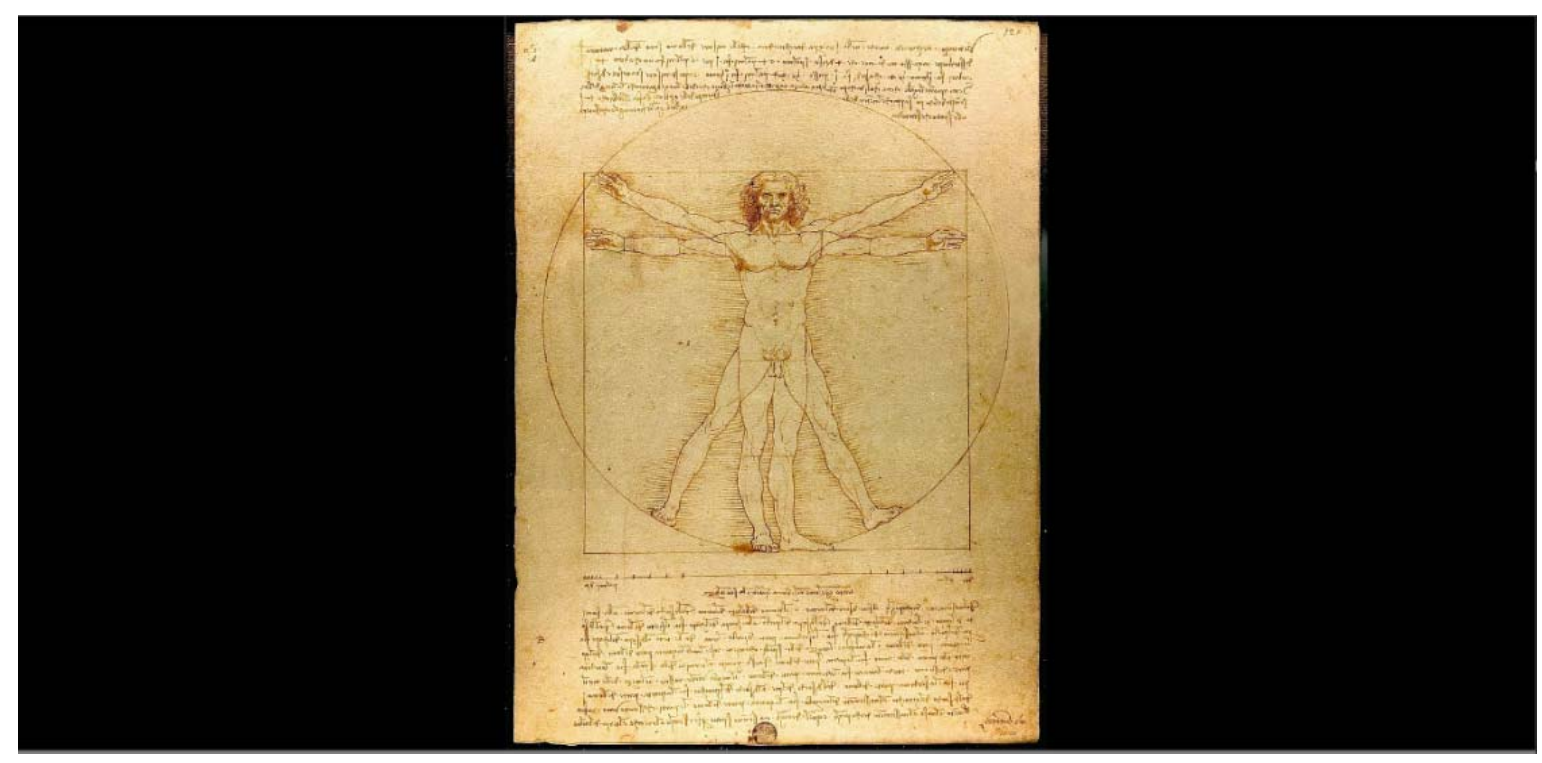

Figure15. Main screen intro, "Vitruvian Man".

The intro starts playing and an old man starts mumbling Joseph Merrick's version of "False Greatness" by Isaac Watts while the "Vitruvian Man" by Leonardo Da Vinci appears in the center, this drawing refers to human proportion and geometry to portray that the essence of human existence is a collection of memories. Then the four thematic photos start appearing in the four corners, one 
after another. They are in front of me on a huge overwhelming imaginary wall, which is placed too close to trigger discomfort since it is in my personal space.
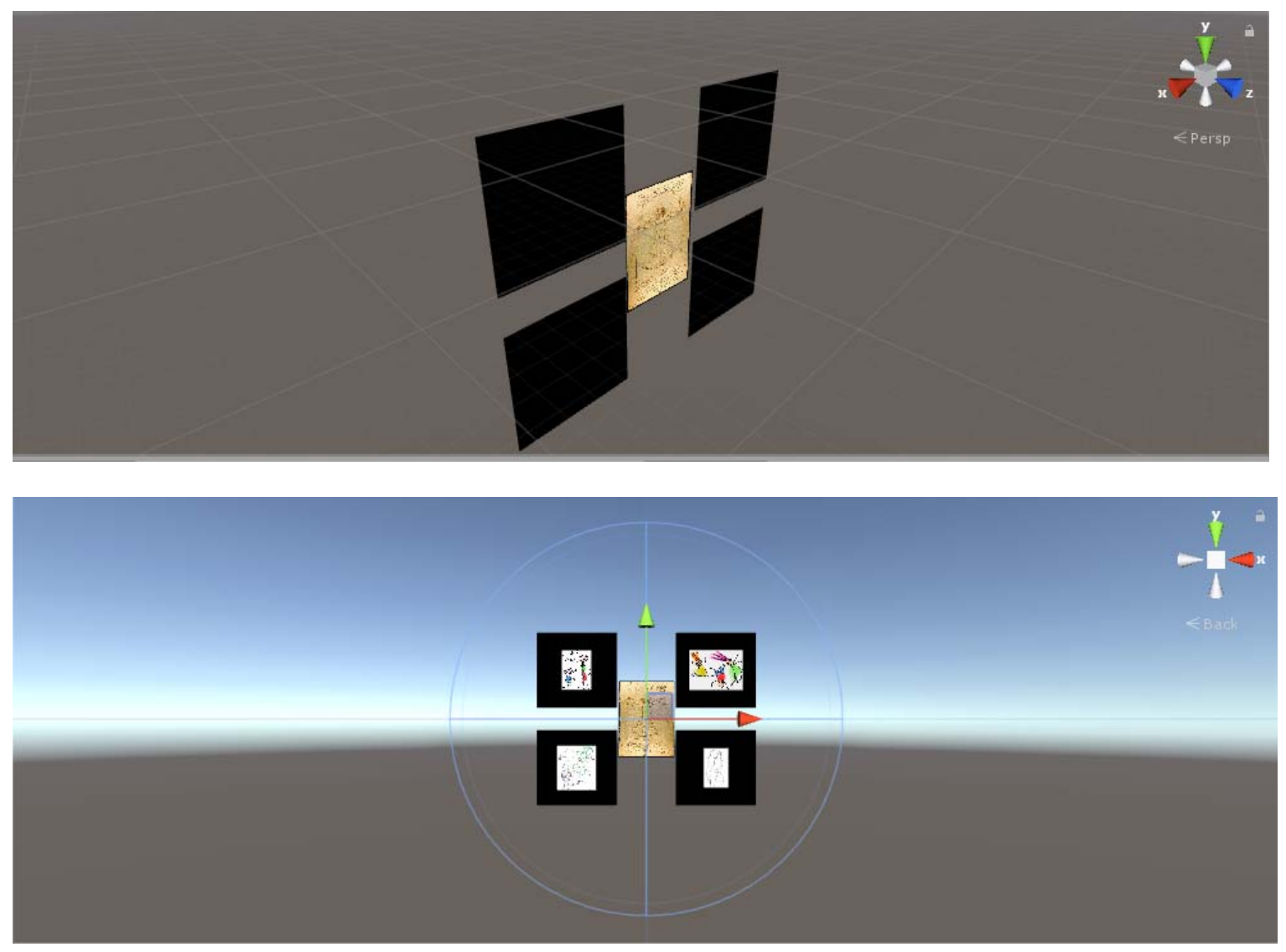

Figure 16. Main screen layout and perspective.

The layout is inspired by the term "The writings on the wall". If I look at one of the four pictures the main screen disappears and a timeline (series of photos and sounds) starts playing. All the sounds are binaural and abstract which triggers my personal memories and related emotions. This increases the emotional effect, based on emotional triggers techniques in method acting. All the photos are drawn into existence as I hear the sound of pencil scribbling on paper. As soon as the timeline finishes, the main screen appears again without the photo at which I have already looked. A noise related to this memory starts playing. I keep experiencing the rest of the photos until I look at all of them. I am left 
with mixed noises related to these thematic photos. Since I am experiencing them in a different order every time, hypothetically, I am left with a different individualized emotional state based on the memories triggered and the sequence of occurrence.
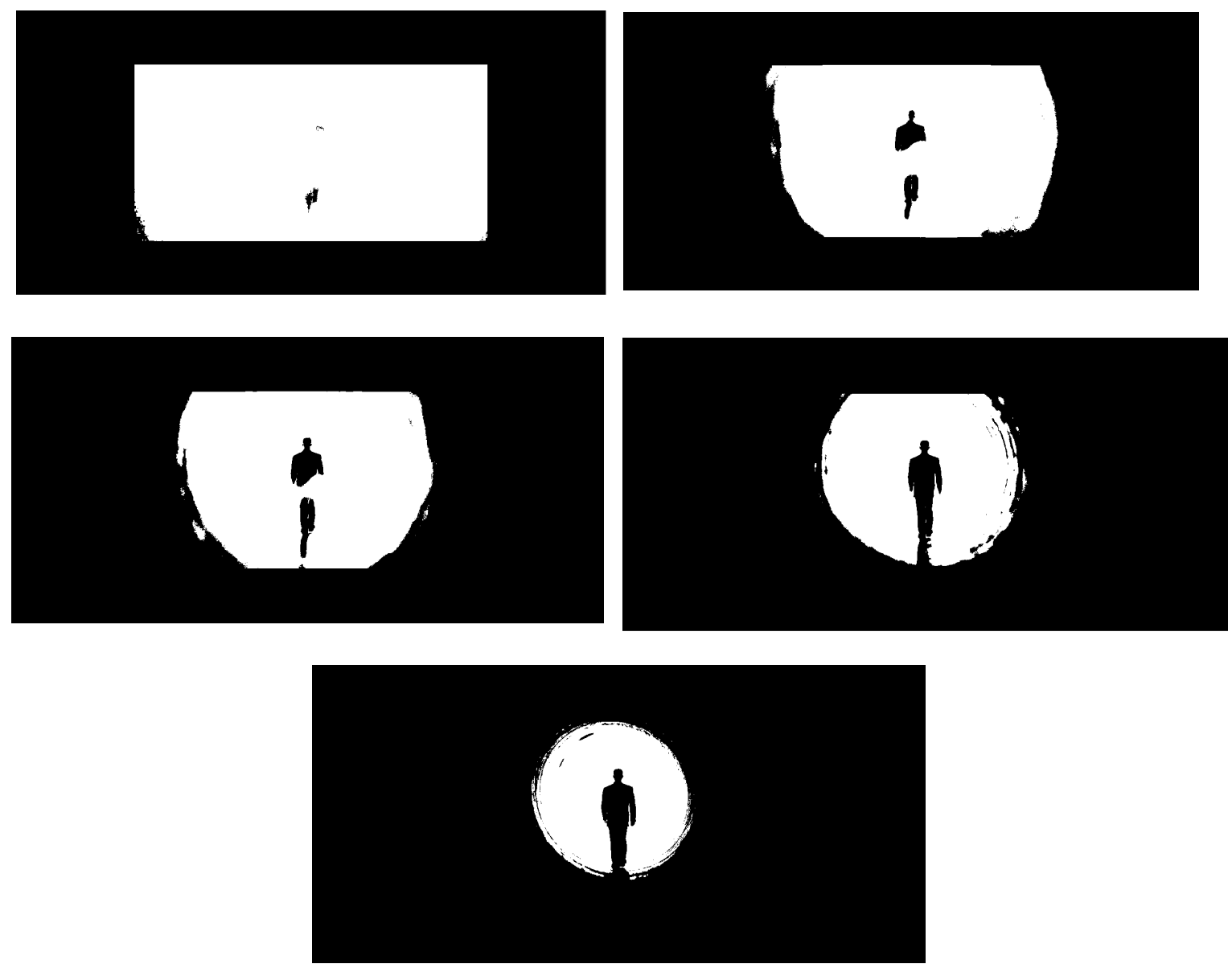

Figure 17. Death tunnel being drawn in the "Temporariness" timeline while the participant hears a binaural recording of a prayer at an ancient church.

One of the moments that stand out is the one in "Temporariness" timeline in figure 17. This moment was described as one of the most powerful in a casual observation. The participants expected to see Jesus or an angel by looking up due to the sound design. It highlighted how impactful spatial sound could be when used with the right queues. 

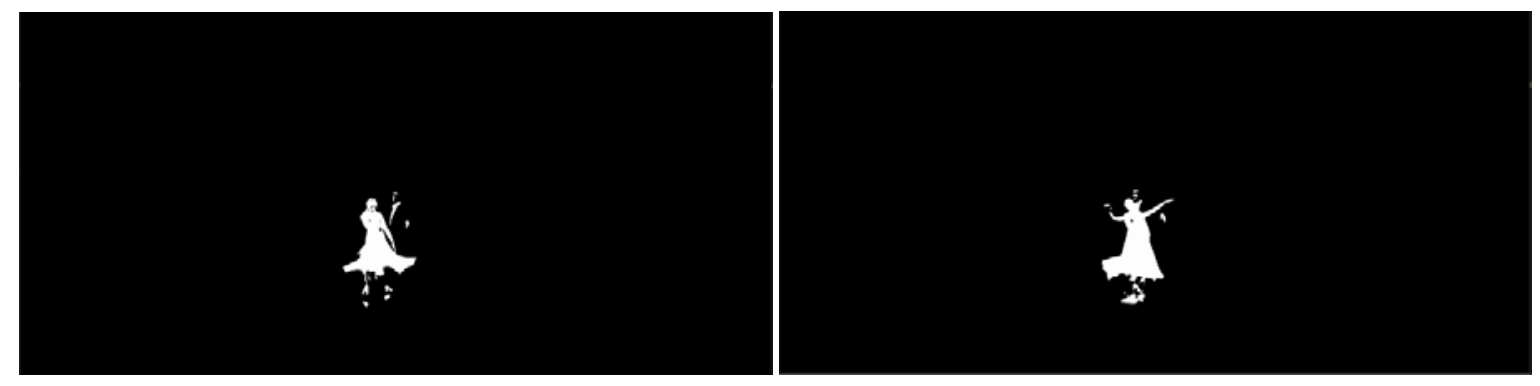

Figure 18. Dancers in the "Sensual" timeline.

Another powerful moment was the dance of the drawing in the "Sensual" timeline. After the dancers are drawn they start dancing around the participant.

In general, the sound and visual cues are a mix of abstract sounds and drawings that trigger personal universal emotions and memories, such as mother cuddling her baby, kids playing at the park and laughing ...etc. These act as memory stimulators and emotional triggers that utilize participants own individual wealth of memories to emphasize the individualized form of experience.

\subsection{Alzheimer's eyes Challenge}

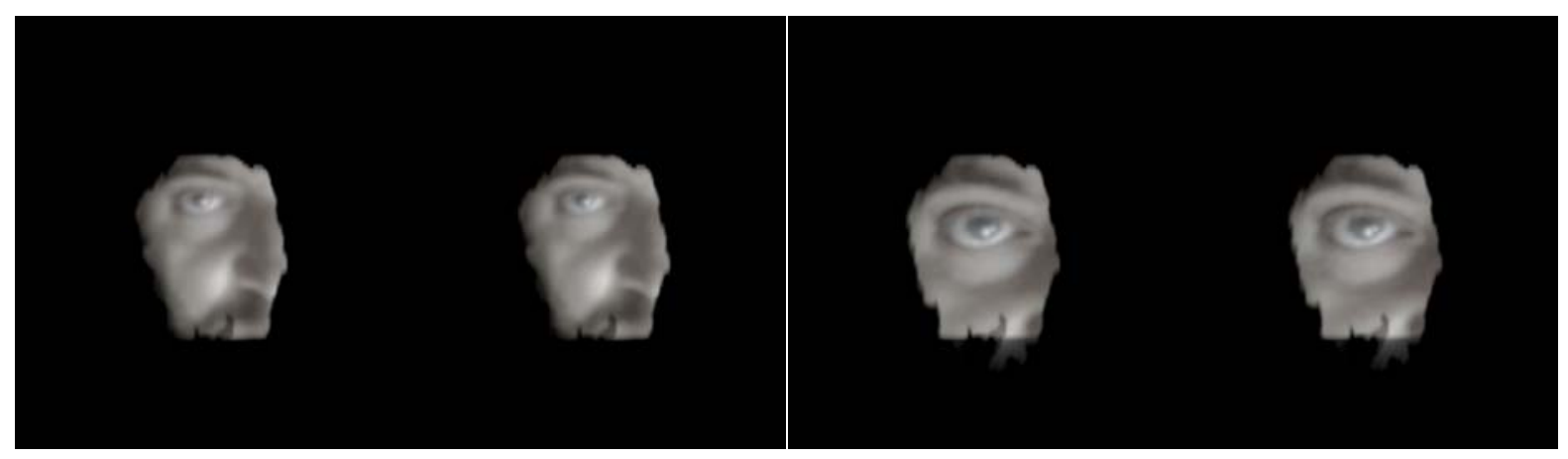

Figure 19. promotional shots taken using the actual experience through the headset.

\subsubsection{The concept}

One of the experiences is "Alzheimer's Eyes Challenge"; a mixed reality experience that allows the participants to experience some of the symptoms of Alzheimer's in the form of serious games. The way 
Alzheimer's patients perceive the world around them is different than the rest of us. And only by stepping into their shoes, we could claim that we are well informed on the condition. Gamifying the notion of the "empathy machine" increases its social impact.

\subsubsection{Detailed Concept}

The intervention takes the form of a challenging user-experience designed with immersion and high-level production values in mind.

This mixed reality project uses a mobile-handset-based head-mounted display (MMD) to manipulate the user's view of the world with real-time visual filters; sound-isolating headphones isolates surrounding sound and manipulate it. The project is built in Unity, using the Vuforia plug-in for real-time video manipulation, and custom audio-filters for sound manipulation. In a fashion that simulates Alzheimer's disease symptoms, visual and auditory senses are manipulated to, we hope, influence the participant's cognitive and emotional experience of the real world.

Our desired user-journey takes the form of the following figure:

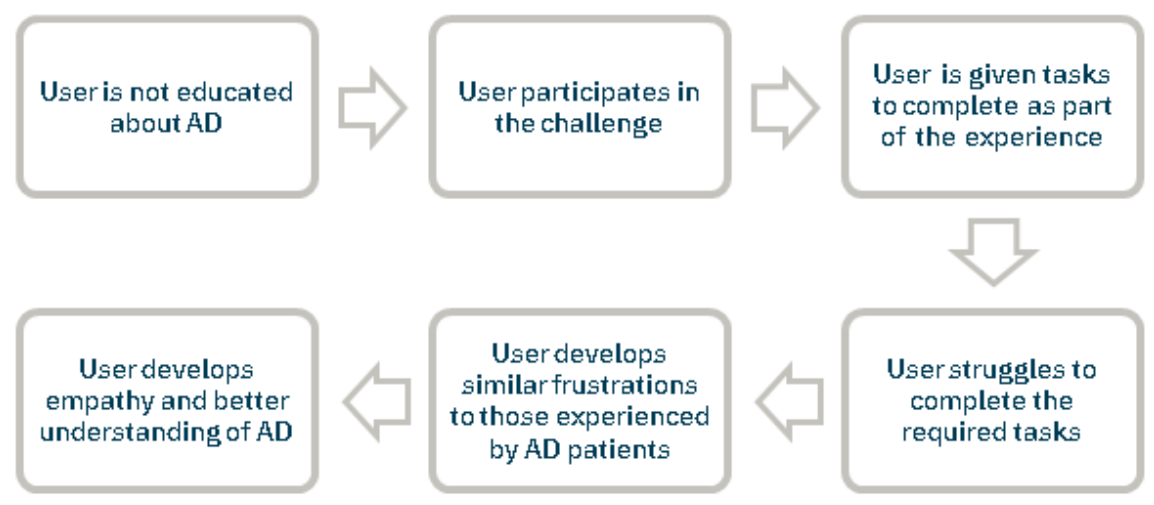

Figure 20. The challenge scenario and desired outcome.

\subsubsection{Types of challenges}

The game-like experience challenges the user through three types of challenges: 
The first Type is a single-participant mode, in which the participant goes through 6 tasks; each task is accompanied by the simulation of symptoms that mimic the progression of $\mathrm{AD}$ and its effect on the way $\mathrm{AD}$ patients experience the world. The experience starts with a health and safety warning; the user then dons the headset/headphones. They move between six physical, real-world stations with a task to complete at each station. Each station activates different visual and auditory filters using the same MR technology.

The second Type is a multi-participant mode, in which two or more participants go through the same six stages challenge, with one participant not wearing the headset. The game condition invites each participant to finish as fast as possible; the winner is the one who finishes first.

The third Type is also a multi-participant mode. Two or more participants are asked to experience the world in a social context, while wearing the headset. They will have to interact with each other and interview five people in the setting. This includes interviewing a maximum of four participants who are wearing the headset. The interview will consist of 5 questions on which they will have to report by the end from memory. The goal is to evaluate how effectively they could communicate while experiencing the significant sensory hurdles of the mixed reality application.

\subsubsection{Target audience}

We approach the design from different potential user points of view:

o Patients: by helping them understand and set expectations and progression.

o Patient's caregivers: by providing them with proper training through simulation which is a big field in nursing education.

o Patient's family members: to understand the reasons behind their relative's actions.

o Healthcare students: to understand practically how it feels to have Alzheimer's disease instead of reading about it.

o Method actors: to build authentic characters around this disease. 
o Volunteers and social service enthusiasts: to build empathy and promote meaningful awareness.

o Digital Media enthusiasts: to promote the potential of technology for social good.

o Gamification enthusiasts: to promote the potential of gamification for social good.

Although these different categories don't require unique versions of the challenge at this stage, the need to build different versions might arise as the challenge develops further. Furthermore, it is worth mentioning that, for precautionary reasons, like any new extended reality experience, it is not recommended to allow people with epilepsy or similar conditions to take the challenge until it is verified medically to be safe for them by our clinical end-user advisors/ co-designers.

\subsubsection{Selected participants' profiles and Scenarios:}

o Alzheimer's patients' caregivers are hired by the seniors' center, they are asked to participate in the experience which is organized, as part of their training, to develop a better understanding of their patients, after the experience the caregivers are more capable of handling these cases based on the knowledge and empathy acquired.

o Alzheimer's patient's family members, once their relative is diagnosed with Alzheimer's, are asked by the doctor to go through the experience to better understand the suffering of their relative. After going through the experience, the family develops a sense of empathy towards their relative and become more forgiving of their actions.

o Healthcare students are studying Alzheimer's disease as part of their curriculum; their professor asks them to sign up for the experience. They go through it to understand practically how it feels to suffer from Alzheimer's; by the end of the experience, their education is more efficient because they have moved from reading about the condition to experiencing it. This would be the manifestation of problem-based learning or hands-on learning. 


\subsubsection{Similar experiences}

After researching, we found several attempts at building experiences that imitates Alzheimer's and Dementia as conditions using analog and extended reality platforms such as:

o A Walk through Dementia (2018): it is a virtual reality experience developed by Alzheimer's Research UK.

o Virtual Dementia Tour (2010): It is a non-technology based twelve minutes tour, developed by Second Wind Dreams founder, P.K. Beville, protected by patent (U.S. No. 8,388,347).

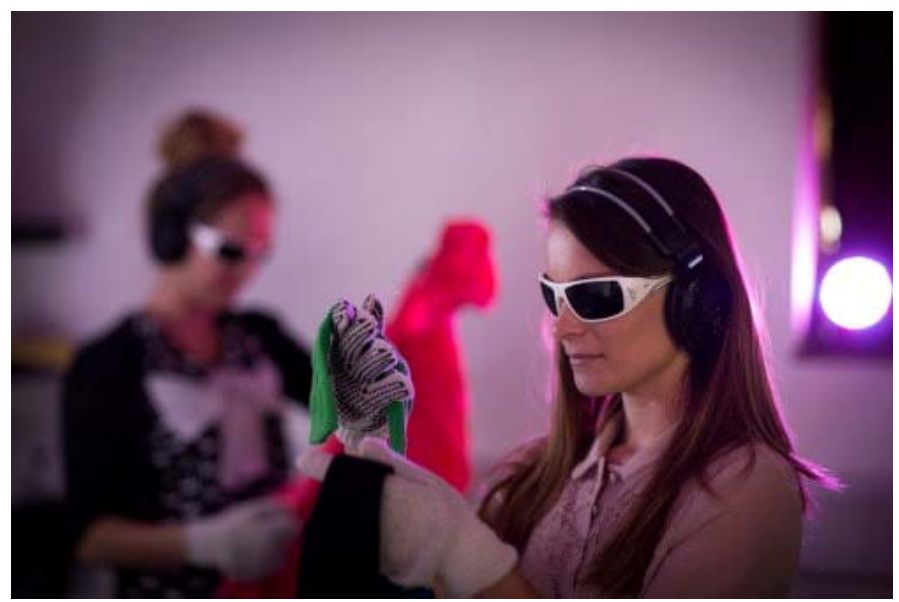

Figure 21. Virtual Dementia Tour (Second Wind Dreams, 2018)

o Virtual Dementia Experience (2014): An award-winning virtual experience by Opaque Multimedia and Alzheimer's Australia Vic, which claims to be a pioneering effort in the field of "empathetic education".

However, we could confidently argue that Alzheimer's Eyes Challenge, has the potential to increase efficiency and realism in its delivery based on our observations and design approach. As shown in the following comparison table: 


\begin{tabular}{|c|c|c|c|c|}
\hline Experience Name & $\begin{array}{c}\text { Virtual Dementia } \\
\text { Tour }\end{array}$ & $\begin{array}{l}\text { A walk Though } \\
\text { Dementia }\end{array}$ & $\begin{array}{l}\text { Virtual Dementia } \\
\text { Experience }\end{array}$ & $\begin{array}{l}\text { Alzheimer's Eyes } \\
\text { Challenge }\end{array}$ \\
\hline Platform & Analog & Virtual Reality & VR / Flat screen & Mixed Reality \\
\hline Format & Tasks / Tour & Storytelling & $\begin{array}{c}\text { Storytelling / } \\
\text { Tour }\end{array}$ & $\begin{array}{c}\text { Gamified } \\
\text { Challenge }\end{array}$ \\
\hline Structure & Number of tasks & Scenario & Scenario & $\begin{array}{l}\text { Sequence of tasks } \\
\text { to resemble } \\
\text { Alzheimer's } \\
\text { progression } \\
\text { (Potentially) }\end{array}$ \\
\hline Environment & Real world & 3D / 360 film & 3D modeling & Real world \\
\hline Time-location & Real time & Scenario based & Scenario based & Real time \\
\hline Scenarios & 1 & 3 daily routines & 1 & $\begin{array}{l}3 \text { types of } \\
\text { challenges }\end{array}$ \\
\hline Context & Individual & Individual & Individual & $\begin{array}{l}\text { Social and } \\
\text { Individual }\end{array}$ \\
\hline Interactivity & Analog & $\begin{array}{c}\text { Google } \\
\text { Cardboard App }\end{array}$ & Interactive & Interactive \\
\hline Embodiment & Embodied & Less Embodied & Less Embodied & Embodied \\
\hline Purpose & Training & Awareness & $\begin{array}{l}\text { Empathetic } \\
\text { Education }\end{array}$ & Multi Purpose \\
\hline Equipment & $\begin{array}{l}\text { Goggles, gloves, } \\
\text { stones in shoes, } \\
\text { headphones and } \\
\text { player }\end{array}$ & $\begin{array}{l}\text { VR headset and } \\
\text { headphones }\end{array}$ & $\begin{array}{l}\text { VR headset / } \\
\text { screen and } \\
\text { headphones }\end{array}$ & $\begin{array}{l}\text { Phone based } \\
\text { headset and } \\
\text { headphones }\end{array}$ \\
\hline Manipulation & $\begin{array}{l}\text { Visual, auditory } \\
\text { and physical }\end{array}$ & Visual & Visual & $\begin{array}{c}\text { Visual and } \\
\text { auditory as a start }\end{array}$ \\
\hline $\begin{array}{l}\text { Manipulation } \\
\text { format }\end{array}$ & $\begin{array}{l}\text { Fixed manipulation } \\
\text { (One for each } \\
\text { sense) in flexible } \\
\text { scenarios }\end{array}$ & $\begin{array}{l}\text { Fixed (Multi } \\
\text { options) in a } \\
\text { fixed scenario }\end{array}$ & $\begin{array}{l}\text { Fixed (Multi } \\
\text { options) in a } \\
\text { fixed scenario }\end{array}$ & $\begin{array}{c}\text { Fixed (Multi } \\
\text { options) in flexible } \\
\text { scenarios }\end{array}$ \\
\hline Fidelity & $\begin{array}{l}\text { Medium, although } \\
\text { it is in real time, } \\
\text { the auditory } \\
\text { manipulation is } \\
\text { done by adding } \\
\text { noise only }\end{array}$ & $\begin{array}{l}\text { Low because it is } \\
\text { a pre-set scenario }\end{array}$ & $\begin{array}{l}\text { Low because it is } \\
\text { a pre-set scenario }\end{array}$ & $\begin{array}{l}\text { Medium with the } \\
\text { possibility to } \\
\text { increase, relatively, } \\
\text { through testing. } \\
\text { Auditory } \\
\text { manipulation is } \\
\text { real. }\end{array}$ \\
\hline Fidelity issues & $\begin{array}{c}\text { Embraces the } \\
\text { medium's fidelity } \\
\text { issues }\end{array}$ & $\begin{array}{l}\text { Embraces the } \\
\text { medium's } \\
\text { fidelity issues }\end{array}$ & $\begin{array}{l}\text { Embraces the } \\
\text { medium's } \\
\text { fidelity issues }\end{array}$ & $\begin{array}{c}\text { Tries to } \\
\text { incorporate and } \\
\text { benefit from the } \\
\text { medium's fidelity } \\
\text { issues }\end{array}$ \\
\hline
\end{tabular}

Table 1. Comparison between different Alzheimer's and Dementia experiences 
Based on the comparison table, it is clear that there is an element of novelty, in our challenge, in addition to the potential to serve an important need; however, it is too early to claim that the first prototype will achieve all the desired outcomes. The next phase of testing and maintenance is crucial to perfect the tasks and technology.

Finding similarities with our design concepts, as a result of our research, has confirmed that we are on the right track and helped us avoid duplicating intellectually protected projects.

\subsubsection{The technical design}

\subsubsection{Design Principles}

o This experience is easy to use, immersive, self-explanatory, realistic and challenging to the user.

o This experience is affordable once you acquire the required technology.

o This experience gives a professional feel to it in comparison to the old-fashioned way.

o This experience could get rid of the human factor and become a user experience through preset auto settings.

o This experience is interactive as opposed to any experience available at the moment.

o This experience utilizes the most up-to-date technology, as opposed to the old-fashioned approach used.

\subsubsection{Technical Simulation}

\subsection{Visual aging symptoms:}

These are simulated by using filters (shaders) and color manipulation using post-processing effects in unity. 

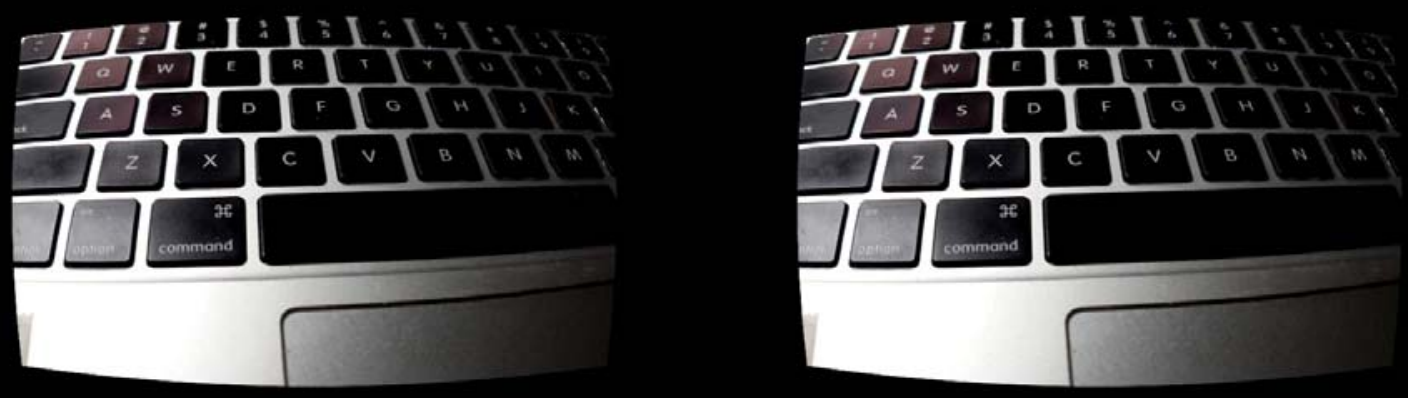

Figure 22. Visual effects through the headset: normal vision.

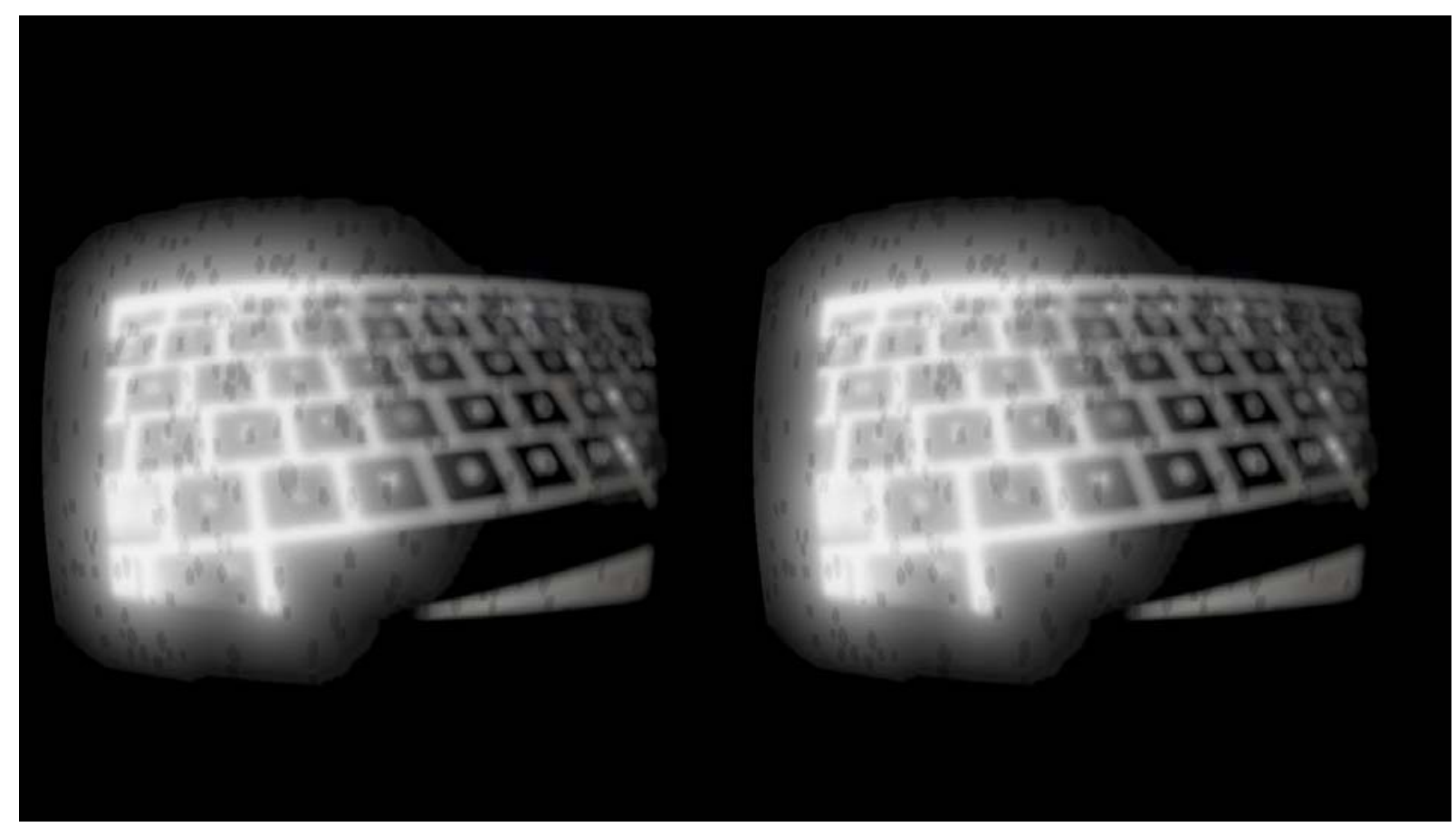

Figure 23. Visual effects through the headset: eye floaters and advanced cataract. 


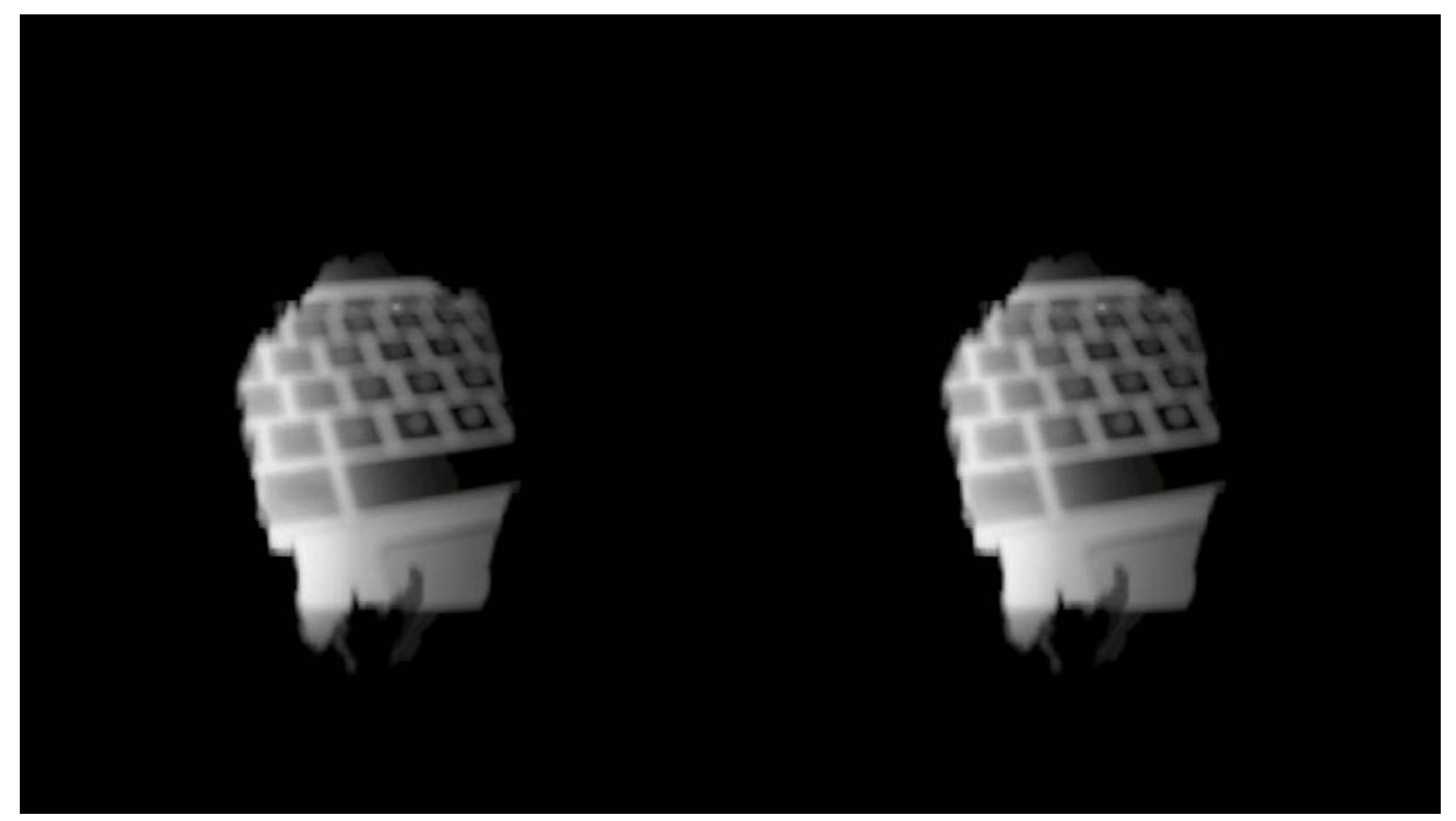

Figure 24. Visual effects through the headset: Macular degeneration and advanced cataract.

To initiate dizziness and nausea in the sixth stage, the display shakes horizontally and vertically. All the visual and auditory effects are built in a way that allows easy customization by entering numerical values or moving sliders. This is one of the main strengths of the design in general.
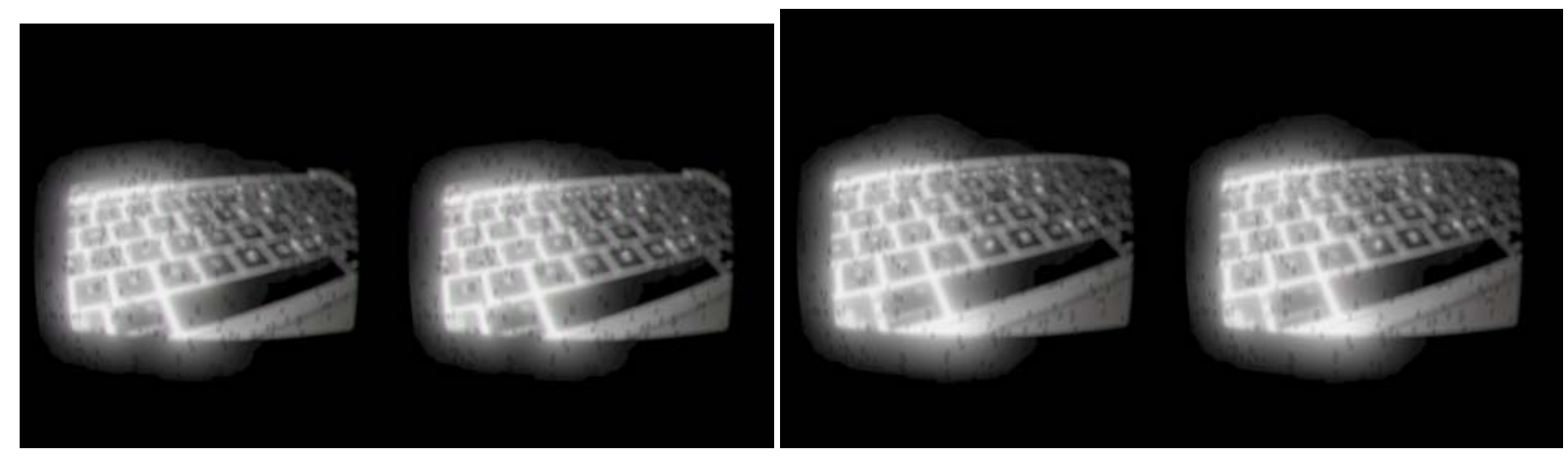

Figure 25. Visual effects through the headset: stage six, eye floaters and shaking display 


\subsection{Hearing aging symptoms:}

These would be simulated by adding different auditory filters such as Radio-like distortion, low and high pass filters, omitting words, tinnitus ring, and binaural sound effects to distract in a way that simulates hallucinations or distracting thoughts.

\subsubsection{Detailed Description of the first type of the challenge}

\subsubsection{Scenario}

- The user will be given different tasks to complete. Each task represents a stage of Alzheimer's progression.

- Each task will differ in nature, one task is solving simple mathematical equations, another task is arranging a table, or identifying certain colour from a group of very close shades of the same colour ...etc.

- There will be stickers around the experience location that would change the filters or create a sudden effect once looked at to mimic the sudden shifts in brain comprehension in patients.

- The total experience will be causing discomfort and stress as the activity will be timed.

- By the end of the experience, the user experience is explained to emphasize the meaningful awareness after building empathy.

\subsubsection{Detailed user flow, addressing any UI required}

- The user flow is described in the tasks details.

- There are no special user interface (UI), everything should be realistic in order not to lose the experience's immersive nature. 


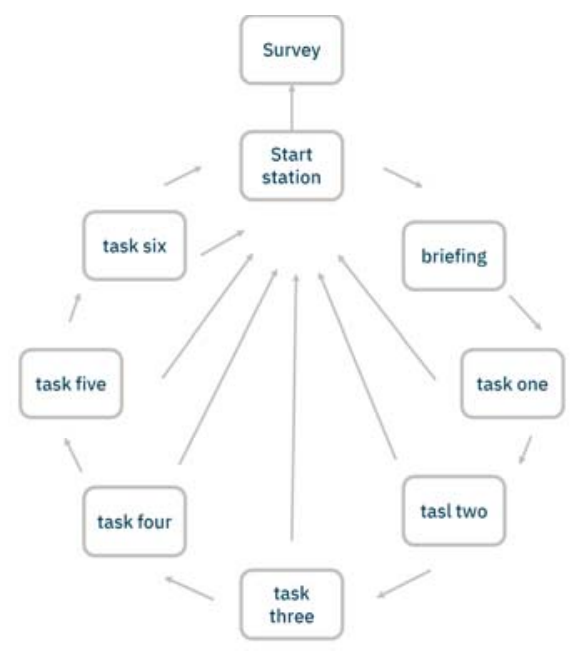

Figure 26. Alzheimer's Eyes Challenge User Flow Chart.

\subsection{General attributes of the experience:}

- Events are triggered by images (could be triggered by shapes as well).

- Every 2 minutes the participant will be reminded of how much time has already passed. Like a quarter has passed, half the time has passed ...etc. (this is an upgrade in the next version which is meant to increase pressure).

- The total length of the experience is ten minutes from start to end.

- To escape the experience the participant should go to the start station and look at the escape sign to exit and start the relaxation experience.

- To finish the experience after going through all tasks the participant should go back to the beginning of the experience and look at the success sign.

- If the participant gives up, the participant is given verbal reinforcement by the challenge supervisor to highlight the importance of supportive attitude. 

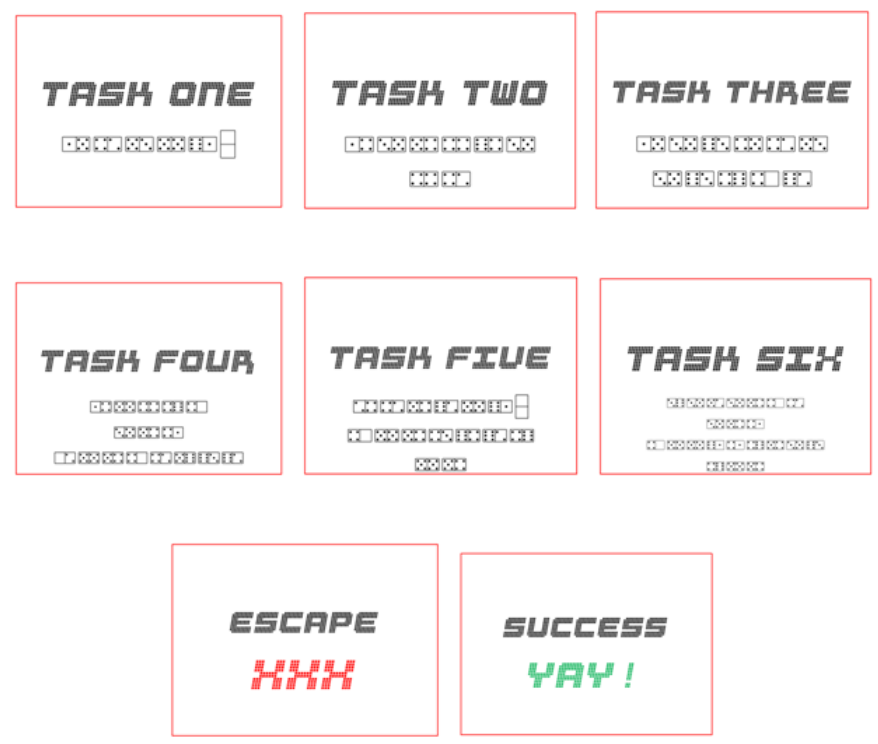

Figure 27. The exact images which should be recognized by Vuforia to trigger different stages.

\subsection{Refined experience prototype design and sequence:}

Ideally, the prototype should be structured as described below. However, due to some technical difficulties, task six and the augmented survey have been delayed to a later stage and the prototype was downscaled. Additionally, the filters for each task are going to be modified based on the user experience evaluation procedures in the next phase of this research.

The experience would be compromising of six tasks, to be achieved in ten minutes using the auditory and visual filters that we mentioned earlier.

\section{Task 1: Memory}

- $\quad$ The stress evoking beats will start at speed 1 .

- $\quad$ The Actual Task (samples):

- Memorize several things and write them down.

- $\quad$ Remember 5 sentences and write them down.

- Writing down the 7-8 digit numbers you hear. 


\section{Task 2: Language}

- $\quad$ The stress evoking beats will play at speed 2 .

- The Actual Task (samples):

- $\quad$ Solve a small puzzle of $3 \mathrm{X} 3$ words on paper.

- Read text on paper.

\section{Task3: Mathematics}

- $\quad$ The stress evoking beats will play at speed 3.

- The Actual Task (samples):

- Solve five simple equations or mathematical problems on a piece of paper.

\section{Task 4: Logic and Concepts}

- $\quad$ The stress evoking beats will play at speed 4 .

- $\quad$ The Actual Task (samples):

- Look at the sign and read out loud a small logical problem $\backslash$ concept $\backslash$ then write down the solution.

\section{Task 5: Sensory Confusion}

- $\quad$ The stress evoking beats will play at speed 5 .

- Auditory Filters: the main part is the emotional music then happy music at short intervals will play in the background to stimulate the mood swings, radio, and hallucinations with tinnitus.

- The Actual Task (samples):

- Pick the bright red or orange t-shirt from a pile of clothes.

- Match six pairs of colorful socks.

\section{Task 6: balance and coordination}

- $\quad$ The stress evoking beats will play at speed 6 .

- The Actual Task (samples):

- Walk on a straight line or in a corridor. 
- Carry a box to move it from one side to another.

7. The final survey (this is delayed to the next version, for now, it is just verbal questions for self-reflection):

- This is triggered by escape or success images:

- However, it could be delivered in one of two forms:

- $\quad$ Paper form.

- Electronic form as part of the experience.

\section{The recovery experience:}

- This is the final stage to eliminate the emotional distress that was caused by the experience.

- Consists of:

- Happy music.

- Possibly Affirmations.

- Possibly positive images that trigger happy emotions.

\section{Discussion and conclusions}

The initial feedback on "Silenced Memories" the virtual reality experience is that it is a true experience. The participants found it enjoyable and didn't come up with conclusions but rather an emotional impact that couldn't be articulated.

On the other hand, Alzheimer's eyes challenge has highlighted that gamified mixed reality experiences could build effective empathy machines and fulfill technology for good concept, i.e. media ethical and social responsibility. This research is very multidisciplinary in nature and would serve a big sector of the society. Its importance lies in the fact that it could keep expanding to support bigger sectors of the society, through its utility use.

Furthermore, aworking prototype of the mobile application, that constitutes the core of Alzheimer's Eyes challenge, has been developed. Debugging and tests are being conducted to ensure its effectiveness. 
Additionally, a bank of tasks is being created to be used in constructing different challenges based on the same model.

No formal surveying is going to be conducted in the first run at the showcase. However, general observations will be conducted to develop the challenge further, and to help design a formal study on the experience of users, and changes in their self-assessment of empathy using existing instruments from psychological research. The precise instruments will be determined as the research project progresses.

\subsection{Potential for innovation}

\subsubsection{Social and technological innovation}

Alzheimer's eyes challenge has potential for social and technological innovation through its unique utilization of mixed reality technology. The true novelty in this challenge is not a result of using new technology or concepts but rather utilizing existing modern technology and the concepts of serious games and empathy machines to build a novel user experience design. This design aims to create a challenge that is entertaining and fosters learning and stimulates empathy and emotions through immersive design. As a result, it could be used as a tool to increase the inclusivity of seniors suffering from Alzheimer's disease, as well as providing a powerful training tool to all stakeholders.

\subsubsection{Policy and practice innovation}

Knowledge mobilization is a major element in this project. As we progress in this research we accumulate a list of recommended design guidelines that could be utilized in further research. These could become the basis for the next step in the experience development and study of its utility use.

\subsection{Implementation issues}

o When building augmented reality experiences in Unity, there are some stability issues on mobile phones. 
o The augmented reality application had to be re-engineered several times to work properly. This is due to the lack of programming experience and mobile job management support in previous unity versions.

o Audio filters have proven to be very demanding on mobile phones' memory.

o The app is very demanding, on the mobile phones, which requires faster mobile phones. It will become stronger as unity increases support for multitasking and memory management for mobile phones.

o The learning curve of programming for augmented reality as a platform is very big, but once achieved, it opens a big world of possibilities and applications.

o The level of fidelity is going to keep increasing based on two factors, the technology capabilities, and the medical research that would result in accurate simulation. This would, in turn, increases the realism of the experience and accuracy.

\subsection{Future Directions:}

Designing this challenge went through different stages by researching Alzheimer's disease, empathy machines, serious games and extended realities' (VR, AR, and MR) technologies and affordances solutions to build the final prototype design. This development process was agile and iterative.

The next step will be conducting qualitative research and Think-Aloud Protocols (TAP) to collect meaningful qualitative data from the participants to evaluate the design. These protocols are described by K. Anders Ericsson (2003) as the valid and non-reactive verbalization of thoughts during the performance of tasks. It is a way of collecting raw spontaneous feedback from the participants as they are going through the challenge. This would work perfectly in line with the experience conditions since the participants are encouraged to communicate during the experience to ensure their safety.

On the other hand, there will be a deeper coverage of some very important topics which couldn't be covered in the initial stage such as ethical related concerns and practices in addition to clinical feedback and evaluation. 


\section{References}

A Walk Through Dementia. (n.d.). Retrieved July 12, 2018, from http://awalkthroughdementia.org/

About the brain | Alzheimer Society of Canada. (n.d.). Retrieved July 12, 2018, from http://alzheimer.ca/en/Home/About-dementia/Alzheimer-s-disease/About-the-brain

Adler, S. (1988). The technique of acting. New York: Bantam Books.

$\begin{array}{lllllll}\text { Age-Related } & \text { Eye } & \text { Diseases. } & \text { (n.d.). Retrieved July 12, 2018, from }\end{array}$ https://nei.nih.gov/healthyeyes/aging_eye

Archer, D., \& Finger, K. (2018, March). Walking in another's virtual shoes: Do 360-degree video news stories generate empathy in viewers? Retrieved July 12, 2018, from https://www.cjr.org/tow_center_reports/virtual-reality-news-empathy.php

Barfield, W., \& Hendrix, C. (1995). The effect of update rate on the sense of presence within virtual environments. Virtual Reality, 1 (1), 3-15. doi:10.1007/bf02009709

Barr, T. (1997). Acting for the Camera: Revised Edition. Harper Perennial.

Batson, C. D. (1991). Altruism question: Toward a social psychological answer.

Batson, C. D., Sympson, S. C., Hindman, J. L., Decruz, P., Todd, R. M., Weeks, J. L., . . Burns, C. T. (1996). "Ive Been there, Too": Effect on Empathy of Prior Experience with a Need. Personality and Social Psychology Bulletin, 22 (5), 474-482. doi:10.1177/0146167296225005

Batson, C. D., Early, S., \& Salvarani, G. (1997). Perspective Taking: Imagining How Another Feels Versus Imaging How You Would Feel. Personality and Social Psychology Bulletin, 23 (7), 751-758. doi: $10.1177 / 0146167297237008$ 
Batson, C. D. (2009). These Things Called Empathy: Eight Related but Distinct Phenomena. The Social Neuroscience of Empathy, 3-16. doi:10.7551/mitpress/9780262012973.003.0002

Baudrillard, J. (1995). The Virtual Illusion: Or the Automatic Writing of the World. Theory, Culture \& Society,12 (4), 97-107. doi:10.1177/026327695012004007

Bauman, Z. (2000). Time and Space Reunited. Time \& Society, 9 (2-3), 171-185. doi:10.1177/0961463x00009002002

Bazin, A., \& Andrew, J. D. (2014). André Bazin's new media. Oakland: University of California Press.

Begin, P. (2006). Buñuel, Eisenstein, and the 'Montage of Attractions': An Approach to Film in Theory and Practice. Bulletin of Spanish Studies, 83 (8), 1113-1132. doi:10.1080/14753820601073613

Billinghurst, M., \& Thomas, B. (2011). Mobile Collaborative Augmented Reality. In L. Alem, \& W. Huang, Recent Trends of Mobile Collaborative Augmented Reality Systems. New York, NY: Springer.

Bluebrain | EPFL. (n.d.). Retrieved April 20, 2018, from https://bluebrain.epfl.ch/

Bohart, A. C., \& Greenberg, L. S. (1997). Empathy: Where are we and where do we go from here? Empathy Reconsidered: New Directions in Psychotherapy, 419-449. doi:10.1037/10226-031

Bollmer, G. (2017). Empathy machines. Media International Australia, 165 (1), 63-76. doi:10.1177/1329878x17726794

Brain Tour Part 2. (n.d.). Retrieved July 12, 2018, from https://www.alz.org/alzheimers-dementia/what-isalzheimers/brain_tour_part_2

Bystrom, K., Barfield, W., \& Hendrix, C. (1999). A Conceptual Model of the Sense of Presence in Virtual Environments. Presence: Teleoperators and Virtual Environments, 8 (2), 241-244. doi:10.1162/105474699566107 
Carey, J. (1989). “A Cultural Approach to Communication.” Communication as Culture: Essays on Media and Society. Boston: Unwin Hyman.

Catch Pokémon in the Real World with Pokémon GO! (n.d.). Retrieved April 30, 2018, from http://www.pokemongo.com/

Cavazza, M., Charles, F., \& Mead, S. (2002). Character-based interactive storytelling. IEEE Intelligent Systems, 17 (4), 17-24. doi:10.1109/mis.2002.1024747

Chekhov, M. (1993). On the Technique of Acting.

Dachis, A. (2016, Sep 9). How Virtual \& Mixed Reality Trick Your Brain. Retrieved from Next Reality: https://next.reality.news/news/virtual-mixed-reality-trick-your-brain-0171367/

Decety, J., \& Lamm, C. (2009). Empathy versus Personal Distress: Recent Evidence from Social Neuroscience. The Social Neuroscience of Empathy, 199-214. doi:10.7551/mitpress/9780262012973.003.0016

Delwiche, A. A., \& Henderson, J. J. (2012). The participatory cultures handbook. New York: Routledge.

Dementia symptoms and areas of the brain. (n.d.). Retrieved July 12, 2018, from https://www.alzheimers.org.uk/about-dementia/symptoms-and-diagnosis/how-dementiaprogresses/symptoms-brain

Eisenstein, S. (1991). Selected Works: Towards a theory of montage. BFI Pub.

Elliott, R., Bohart, A. C., Watson, J. C., \& Greenberg, L. S. (2011). Empathy. Psychotherapy Relationships That Work,132-152. doi:10.1093/acprof:oso/9780199737208.003.0006

Elliott, R., Bohart, A. C., Watson, J. C., \& Greenberg, L. S. (2011). Empathy. Psychotherapy Relationships That Work,132-152. doi:10.1093/acprof:oso/9780199737208.003.0006 
Ericsson, K. A. (2003). Valid and Non-Reactive Verbalization of Thoughts During Performance of Tasks Towards a Solution to the Central Problems of Introspection as a Source of Scientific Data. Journal of Consciousness Studies, 10, 1-18.

Gallese, V. (1998). Mirror neurons and the simulation theory of mind-reading. Trends in Cognitive Sciences,2 (12), 493-501. doi:10.1016/s1364-6613(98)01262-5

Glusker, A. (2017). Motivational Design and Problem-Based Learning May Increase Student Engagement in Information Literacy Instruction Sessions. Evidence Based Library and Information Practice,12(4), 259. doi:10.18438/b8pq1k

Grau, O. (2003). Virtual art: From illusion to immersion. Cambridge, MA: MIT Press.

Guynes, S., \& Hassler-Forest, D. (2017). Star Wars and the History of Transmedia Storytelling. Amsterdam: Amsterdam University Press.

Hagen, U., Frankel, H., \& Pierce, D. H. (2009). Respect for Acting. Hoboken: John Wiley \& Sons.

Hearing Loss: A Common Problem for Older Adults. (n.d.). Retrieved July 12, 2018, from https://www.nia.nih.gov/health/hearing-loss-common-problem-older-adults

Heeter, C. (1992). Being There: The Subjective Experience of Presence. Presence: Teleoperators and Virtual Environments, 1 (2), 262-271. doi:10.1162/pres.1992.1.2.262

Isbister, K. (2017). How games move us - emotion by design. Mit Press.

Jenkins, H. (2003). Rewriting Life Transmedia Storytelling. MIT Technology Review.

Kazan, E. (1997). Elia Kazan: A life. New York: Da Capo Press.

Kim, M. J. (2013). A framework for context immersion in mobile augmented reality. Automation in Construction, 33, 79-85. doi:10.1016/j.autcon.2012.10.020 
Kuppers, P. (2003). Disability and contemporary performance: Bodies on edge. London: Routledge.

Meisner, S., Longwell, D., \& Pollack, S. (1987). Sanford Meisner on acting. Place of publication unknown: Vintage Books, a division of Random House.

Merieau-Ponty, M. (1962). Phenomenology of Perception, trans. London: Routledge: Colin Smith.

Michael, D., \& Chen, S. (2011). Serious games: Games that educate, train, and inform. Mason, OH: Course Technology.

Milk, C. (2015). Chris Milk: How virtual reality can create the ultimate empathy machine. Retrieved July 12 , 2018, from https://en.tiny.ted.com/talks/chris_milk_how_virtual_reality_can_create_the_ultimate_empathy_mac hine

Mischel, W. (2004). Towards integrative science of the person. Annual Review of Psychology, 55, 1-22.

Moore, S., \& Logan, J. (1984). The Stanislavski System: The Professional Training of an Actor; Second Revised Edition.

Moss, L. (2005). The intent to live: Achieving your true potential as an actor. New York: Bantam Dell.

Murray, J. H. (1998). Hamlet on the holodeck: The future of narrative in cyberspace. New York: The Free Press.

O'Brien, L. (2013, June 01). Heath Ledgers father reveals dead actors Joker diary written. Retrieved from https://www.independent.co.uk/arts-entertainment/films/news/heath-ledgers-father-reveals-deadactors-joker-diary-written-during-the-dark-knight-8640660.html

Papagiannis, H. (2017). Augmented Human. OReilly Media. 
Pearlman, E. (2015). I, Cyborg. PAJ: A Journal of Performance and Art, 37 (2), 84-90. doi:10.1162/pajj_a_00264

Pereira, G., Brisson, A., Prada, R., Paiva, A., Bellotti, F., Kravcik, M., \& Klamma, R. (2012). Serious Games for Personal and Social Learning \& Ethics: Status and Trends. Procedia Computer Science, 15, 53-65. doi:10.1016/j.procs.2012.10.058

Rossow, A. (2018, February 28). Cyberbullying Taken To A Whole New Level: Enter The 'Blue Whale $\begin{array}{lllll}\text { Challenge'. } & \text { Retrieved } & \text { April } & 30, & 2018,\end{array}$ https://www.forbes.com/sites/andrewrossow/2018/02/28/cyberbullying-taken-to-a-whole-new-levelenter-the-blue-whale-challenge/\#f869312673ec

Rumble, A. C., Lange, P. A., \& Parks, C. D. (2009). The benefits of empathy: When empathy may sustain cooperation in social dilemmas. European Journal of Social Psychology. doi:10.1002/ejsp.659

Sadowski, W., \& Stanney, K. (2002). Presence in virtual environments. In K. M. Stanney, Human factors and ergonomics. Handbook of virtual environments: Design, implementation, and applications (pp. 791-806). Mahwah, NJ: Lawrence Erlbaum Association.

Shamay-Tsoory, S. G. (2009). Empathic Processing: Its Cognitive and Affective Dimensions and Neuroanatomical Basis. The Social Neuroscience of Empathy,215-232. doi:10.7551/mitpress/9780262012973.003.0017

Singer, M. J., \& Witmer, B. G. (1999). On Selecting the Right Yardstick. Presence: Teleoperators and Virtual Environments,8(5), 566-573. doi:10.1162/105474699566486

Slater, M., \& Wilbur, S. (1997). A Framework for Immersive Virtual Environments (FIVE): Speculations on the Role of Presence in Virtual Environments. Presence: Teleoperators and Virtual Environments, 6(6), 603-616. doi:10.1162/pres.1997.6.6.603 
Statistics Canada. (2016). Data tables, 2016 Census. Retrieved July 12, 2018, from https://www12.statcan.gc.ca/census-recensement/2016/dp-pd/dt-td/index-eng.cfm

Tilt Brush. (n.d.). Retrieved July 12, 2018, from http://www.tiltbrush.com/

Virtual Dementia Tour. (n.d.). Retrieved July 21, 2018, from https://www.secondwind.org/virtualdementia-tourreg.html

VIVE Wireless Adapter. (n.d.). Retrieved July 12, 2018, from https://www.vive.com/eu/wireless-adapter/

Westera, W. (2017). How people learn while playing serious games: A computational modelling approach. Journal of Computational Science,18, 32-45. doi:10.1016/j.jocs.2016.12.002

What Happens to the Brain in Alzheimer's Disease? (n.d.). Retrieved July 12, 2018, from https://www.nia.nih.gov/health/what-happens-brain-alzheimers-disease

Youngblood, G. (1970). Expanded Cinema. E.P. Dutton. 